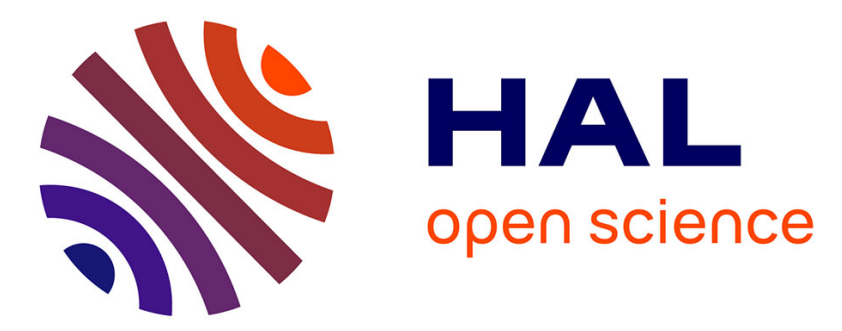

\title{
Guaranteed and fully robust a posteriori error estimates for conforming discretizations of diffusion problems with discontinuous coefficients
}

Martin Vohralík

\section{- To cite this version: \\ Martin Vohralík. Guaranteed and fully robust a posteriori error estimates for conforming discretiza- tions of diffusion problems with discontinuous coefficients. Journal of Scientific Computing, 2011, 46 (3), pp.397-438. 10.1007/s10915-010-9410-1 . hal-00235810v4}

\section{HAL Id: hal-00235810 \\ https://hal.science/hal-00235810v4}

Submitted on 21 Jul 2010

HAL is a multi-disciplinary open access archive for the deposit and dissemination of scientific research documents, whether they are published or not. The documents may come from teaching and research institutions in France or abroad, or from public or private research centers.
L'archive ouverte pluridisciplinaire HAL, est destinée au dépôt et à la diffusion de documents scientifiques de niveau recherche, publiés ou non, émanant des établissements d'enseignement et de recherche français ou étrangers, des laboratoires publics ou privés. 


\title{
Guaranteed and fully robust a posteriori error estimates for conforming discretizations of diffusion problems with discontinuous coefficients*
}

\author{
Martin Vohralík \\ UPMC Univ. Paris 06, UMR 7598, Laboratoire Jacques-Louis Lions, F-75005, Paris, France \\ $\&$ \\ CNRS, UMR 7598, Laboratoire Jacques-Louis Lions, F-75005, Paris, France \\ e-mail: vohralik@ann.jussieu.fr
}

Key words: finite volume method, finite element method, finite difference method, discontinuous coefficients, harmonic averaging, a posteriori error estimates, guaranteed upper bound, robustness

\begin{abstract}
We study in this paper a posteriori error estimates for $H^{1}$-conforming numerical approximations of diffusion problems with a diffusion coefficient piecewise constant on the mesh cells but arbitrarily discontinuous across the interfaces between the cells. Our estimates give a global upper bound on the error measured either as the energy norm of the difference between the exact and approximate solutions, or as a dual norm of the residual. They are guaranteed, meaning that they feature no undetermined constants. (Local) lower bounds for the error are also derived. Herein, only generic constants independent of the diffusion coefficient appear, whence our estimates are fully robust with respect to the jumps in the diffusion coefficient. In particular, no condition on the diffusion coefficient like its monotonous increasing along paths around mesh vertices is imposed, whence the present results also include the cases with singular solutions. For the energy error setting, the key requirement turns out to be that the diffusion coefficient is piecewise constant on dual cells associated with the vertices of an original simplicial mesh and that harmonic averaging is used in the scheme. This is the usual case, e.g., for the cell-centered finite volume method, included in our analysis as well as the vertex-centered finite volume, finite difference, and continuous piecewise affine finite element ones. For the dual norm setting, no such a requirement is necessary. Our estimates are based on $\mathbf{H}$ (div)-conforming flux reconstruction obtained thanks to the local conservativity of all the studied methods on the dual grids, which we recall in the paper; mutual relations between the different methods are also recalled. Numerical experiments are presented in confirmation of the guaranteed upper bound, full robustness, and excellent efficiency of the derived estimators.
\end{abstract}

\section{Introduction}

We consider in this paper a model diffusion problem

$$
\begin{aligned}
-\nabla \cdot(a \nabla p)=f & \text { in } \Omega, \\
p=0 & \text { on } \partial \Omega,
\end{aligned}
$$

*This work was supported by the GNR MoMaS project "Numerical Simulations and Mathematical Modeling of Underground Nuclear Waste Disposal", PACEN/CNRS, ANDRA, BRGM, CEA, EdF, IRSN, France. 
where $\Omega \subset \mathbb{R}^{d}, d=2,3$, is a polygonal (polyhedral) domain (open, bounded, and connected set), $a$ is a scalar diffusion coefficient, and $f$ is a source term. We shall derive here a posteriori error estimates for continuous piecewise affine finite element, vertex-centered finite volume, cell-centered finite volume, and finite difference approximations of this problem.

A posteriori error estimates for finite element discretization of (1.1a)-(1.1b) have been a popular research subject starting from the Babuška and Rheinboldt work [8]. One may formulate the following five properties describing an optimal a posteriori error estimate: 1) deliver an upper bound on the error in the numerical solution which only uses the approximate solution and which can be fully, without the presence of any unknown quantities, evaluated (guaranteed upper bound); 2) give an expression for the estimated error locally, for example in each element of the computational mesh, and ensure that this estimate on the error represents a lower bound for the actual error, up to a generic constant (local efficiency); 3) ensure that the effectivity index, given as the ratio of the estimated and actual error, goes to one as the computational effort goes to infinity (asymptotic exactness); 4) guarantee the three previous properties independently of the parameters and of their variation (robustness); 5) give estimators which can be evaluated locally (negligible evaluation cost). Property 1) allows to give a certified error upper bound, 2) is crucial for the suitability of the estimates for adaptive mesh refinement, 3) and 4) ensure the optimality of the upper bound, and 5) guarantees that the evaluation cost will be much smaller than the cost required to obtain the approximate solution itself.

A vast amount of books and papers have been dedicated to a posteriori error estimates for finite elements. We cite in particular the books by Verfürth [54], Ainsworth and Oden [3], Neittaanmäki and Repin [41], and Repin [47], cf. also Braess [13]. Among the different types of estimators, the so-called equilibrated fluxes estimates, based on equilibration of side fluxes and construction of an $\mathbf{H}$ (div)-conforming flux, enable under certain circumstances to deliver a guaranteed upper bound. These type of estimates are pursued, e.g., by Ladevèze [36], Ladevèze and Leguillon [37], Repin [46], Destuynder and Métivet [24], Luce and Wohlmuth [39], Ainsworth [2], Vejchodský [53], Korotov [35], or Braess and Schöberl [15], and can be traced back to the Prager-Synge equality [44] and the hypercircle method, cf. Synge [51], see also Haslinger and Hlaváček [33], Vacek [52], Nečas and Hlaváček [40], and Hlaváček et al. [34]. They have also recently been shown robust with respect to the polynomial degree in [14]. Much fewer results are known for finite volume methods; we refer, e.g., to $\mathrm{Xu}$ et al. [61] and the references therein.

One particular issue is the robustness with respect to discontinuous coefficient $a$. Robust estimates have been derived by Dörfler and Wilderotter [25], Bernardi and Verfürth [12], Petzoldt [43], Ainsworth [1], or Chen and Dai [23]. All these estimates are, however, based on the "monotonicity around vertices" condition on the distribution of the diffusion coefficient ([12, Hypothesis 2.7]) or a similar assumption. This condition is, unfortunately, very restrictive and in particular excludes the physically interesting cases where regions with different diffusion coefficients meet in a checkerboard pattern and where the weak solution can present singularities. Recently, Cai and Zhang [17] claimed that their estimates do not need any such a condition. This is certainly true for the error upper bound, but [12, Hypothesis 2.7] is still used in [17, Section 4.1] in the lower bound proof.

We try to give in this paper estimates which are as close as possible to the optimality in the sense of the five above properties. Our main purpose is to present estimates which are fully robust with respect to the discontinuities in $a$, and this without the "monotonicity" condition. We are able to achieve this in two different ways. The first one needs the harmonic averaging to be used in the scheme definition, while simultaneously aligning the discontinuities of the diffusion coefficient $a$ with a dual mesh formed around vertices; it uses the energy norm. It is based on the observation of [27] that harmonic weighting can yield robustness in a posteriori error estimates. The second way applies to any method of this paper and requires no alignment of the discontinuities and no use 
of particular averages; it is based on the introduction of a (nonlocal and not locally computable) dual norm, the dual norm of the residual. Such an approach has been pursued by Angermann [4] or Verfürth [55] in the context of robust estimates for convection-diffusion problems and by Chaillou and Suri $[19,20]$ and in $[26]$ in the context of monotone nonlinear problems.

None of the approaches of the present paper gives robust a posteriori error estimates for the standard finite element method with the discontinuities aligned with the original computational mesh elements and with the error measured in the energy norm. We also by no means claim that the two robust approaches are the only and the best possibilities. We rather present them as two simple ways of obtaining robust estimates in the case of discontinuous coefficients. The first approach of aligning the discontinuities with the dual mesh is rather unusual in the finite element method. Nevertheless, it represents a standard way of handling discontinuous coefficients in the cell-centered finite volume (finite difference) approach. We merely show that suitably interpreting the solution of the standard cell-centered finite volume method with harmonic weighting in a finite element basis gives robust energy norm estimates. The key for the robustness of the second approach is the dual norm which actually does not see the jumps in the coefficients. Estimates in this norm are also only globally, and not locally, efficient. We are, however, persuaded that they are more "physical" than the energy norm estimates (see Remark 4.8 below).

We start the paper with preliminaries in Section 2. We then in Section 3 give a list of several different $H^{1}$-conforming methods and recall some useful relations between them. In Section 4, we sketch an abstract framework, both in the energy and dual norms, show its link to the PragerSynge equality [44], and give our a posteriori error estimates. We then discuss four different ways of defining an equilibrated flux. Section 4 is closed by comparisons of the present technique with the residual, equilibrated residual, averaging, functional, and other equilibrated fluxes estimates. The proofs of the (local) efficiency and robustness are the issue of Section 5. All the developments of Sections 4 and 5 are done in an abstract form, not requiring any particular numerical scheme. We show in Section 6 how the estimate and efficiency results apply to the numerical methods of Section 3. Finally, a collection of numerical experiments is presented in Section 7 and some conclusions are drawn in Section 8.

We consider the homogeneous Dirichlet boundary condition only for the sake of clarity of exposition; general boundary conditions can easily be taken in account, as we outline it in [59]. This paper is a detailed description of the results previously announced in [58]; some additional numerical experiments for the finite element method, together with another local minimization strategy, are then studied in [21], and extensions to the reaction-diffusion case in [22].

\section{Preliminaries}

We give in this section the notation and assumptions, recall some important inequalities, and finally give details on the continuous problem (1.1a)-(1.1b).

\subsection{Meshes and notation}

We shall work in this paper with triangulations $\mathcal{T}_{h}$ which for all $h>0$ consist of closed simplices (triangles when $d=2$ and tetrahedra when $d=3$ ) such that $\bar{\Omega}=\bigcup_{K \in \mathcal{T}_{h}} K$. We suppose that the triangulations $\mathcal{T}_{h}$ are conforming (matching), i.e., such that if $K, L \in \mathcal{T}_{h}, K \neq L$, then $K \cap L$ is either an empty set or a common face, edge, or vertex of $K$ and $L$. Let $h_{K}$ denote the diameter of $K$ and let $h:=\max _{K \in \mathcal{T}_{h}} h_{K}$. We denote by $\mathcal{E}_{h}$ the set of all sides of $\mathcal{T}_{h}$, by $\mathcal{E}_{h}^{\text {int }}$ the set of interior, by $\mathcal{E}_{h}^{\text {ext }}$ the set of boundary, and by $\mathcal{E}_{K}$ the set of all the sides of an element $K \in \mathcal{T}_{h} ; h_{\sigma}$ stands for the diameter of a side $\sigma \in \mathcal{E}_{h}$. We also denote by $\mathcal{V}_{h}\left(\mathcal{V}_{h}^{\text {int }}\right)$ the set of all (interior) vertices of 

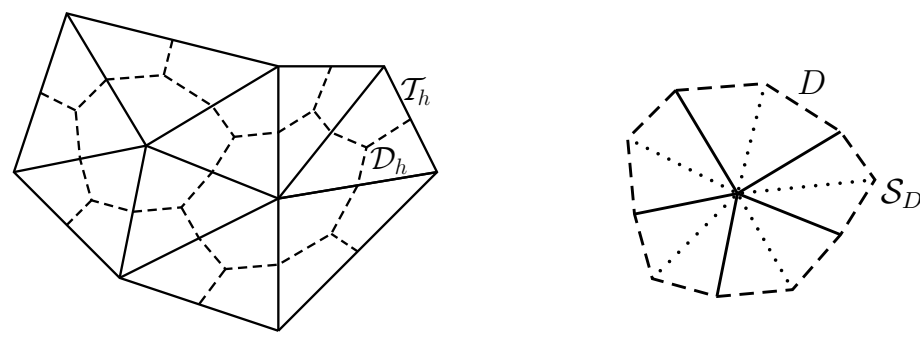

Figure 1: Original simplicial mesh $\mathcal{T}_{h}$ and an associated dual mesh $\mathcal{D}_{h}$ (left) and the fine simplicial mesh $\mathcal{S}_{D}$ of a dual volume $D \in \mathcal{D}_{h}$ (right)

$\mathcal{T}_{h}$. For $V \in \mathcal{V}_{h}$, set $\mathcal{T}_{V}:=\left\{L \in \mathcal{T}_{h} ; L \cap V \neq \emptyset\right\}$, the set of all the elements of $\mathcal{T}_{h}$ which share the given vertex $V$. Similarly, for $K \in \mathcal{T}_{h}$, set $\mathcal{T}_{K}:=\left\{L \in \mathcal{T}_{h} ; L \cap K \neq \emptyset\right\}$, the set of all the elements of $\mathcal{T}_{h}$ which share at least a vertex with the given element $K$.

We shall also consider dual partitions $\mathcal{D}_{h}$ of $\Omega$ such that $\bar{\Omega}=\bigcup_{D \in \mathcal{D}_{h}} D$ and such that for each vertex $V \in \mathcal{V}_{h}, V \in D_{V}$ for exactly one dual volume $D_{V} \in \mathcal{D}_{h}$. The notation $V_{D}$ stands inversely for the vertex associated with a given dual volume $D \in \mathcal{D}_{h}$. We denote by $\mathcal{D}_{h}^{\text {int }}\left(\mathcal{D}_{h}^{\text {ext }}\right)$ the dual volumes associated with the interior vertices from $\mathcal{V}_{h}^{\text {int }}$ (boundary vertices from $\mathcal{V}_{h}^{\text {ext }}$ ), respectively. Next, $\mathcal{F}_{h}$ stands for all sides of the dual partition $\mathcal{D}_{h}$ and $\mathcal{F}_{h}^{\text {int }}\left(\mathcal{F}_{h}^{\text {ext }}\right)$ for all interior (boundary) sides of $\mathcal{D}_{h}$. Let $V \in \mathcal{V}_{h}$. We shall always suppose that $D_{V}$ lies in the interior of the polygon/polyhedron given by $\mathcal{T}_{V}$ (recall that $\mathcal{T}_{V}$ stands for all the simplices sharing the vertex $V$ ). We will also suppose that $\mathcal{E}_{h}^{\text {int }} \cap \mathcal{F}_{h}^{\text {int }}$ has a zero $(d-1)$-dimensional Lebesgue measure. An example of such a partition $\mathcal{D}_{h}$ is given in the left part of Figure 1. In Section 3 below, the meshes $\mathcal{D}_{h}$ will be kept as general as possible. In the other parts of the paper, we will assume that $\mathcal{D}_{h}$ consist of polygonal/polyhedral dual volumes.

In order to define our a posteriori error estimates, we will need a second conforming simplicial triangulation of $\Omega$, that we denote by $\mathcal{S}_{h}$. The basic requirement is that the interiors of the elements of $\mathcal{S}_{h}$ do not intersect the sides of neither the primal partition $\mathcal{T}_{h}$, nor of the dual partition $\mathcal{D}_{h}$. That is, we require $\mathcal{S}_{h}$ to be a conforming refinement of both $\mathcal{T}_{h}$ and $\mathcal{D}_{h}$. For the local efficiency proofs of our estimators, we will later need the assumption that the family of meshes $\left\{\mathcal{S}_{h}\right\}_{h}$ is shape-regular in the sense that there exists a constant $\kappa_{\mathcal{S}}>0$ such that $\min _{K \in \mathcal{S}_{h}} \rho_{K} / h_{K} \geq \kappa_{\mathcal{S}}$ for all $h>0$, where $\rho_{K}$ denotes the diameter of the largest ball inscribed in the element $K$. We will suppose the existence of a local triangulation $\mathcal{S}_{D}$ of each dual volume $D \in \mathcal{D}_{h}$ as shown in the right part of Figure 1 and then set $\mathcal{S}_{h}:=\cup_{D \in \mathcal{D}_{h}} \mathcal{S}_{D}$. We will use the notation $\mathcal{G}_{h}$ for all sides of $\mathcal{S}_{h}$ and $\mathcal{G}_{h}^{\text {int }}\left(\mathcal{G}_{h}^{\text {ext }}\right)$ for all interior (boundary) sides of the partition $\mathcal{S}_{h}$. The notation $\mathcal{G}_{D}^{\text {int }}$ stands for all interior sides of $\mathcal{S}_{D}, \mathcal{G}_{D}^{\text {ext }}$ for all boundary sides of $\mathcal{S}_{D}$, and $\mathcal{G}_{D}$ for $\mathcal{G}_{D}^{\text {int }} \cup \mathcal{G}_{D}^{\text {ext }}$.

Next, for an element $K \in \mathcal{T}_{h}, \mathbf{n}_{K}$ will always denote its exterior unit normal vector; we shall also employ the notation $\mathbf{n}_{\sigma}$ for a unit normal vector of a side $\sigma \in \mathcal{E}_{h}$, whose orientation is chosen arbitrarily but fixed for interior sides and coinciding with the exterior normal of $\Omega$ for boundary sides. For a side from $\mathcal{E}_{h}^{\text {int }}$ shared by $K, L \in \mathcal{T}_{h}$, which we denote by $\sigma_{K, L}$, such that $\mathbf{n}_{\sigma_{K, L}}$ points from $K$ to $L$ and a function $\varphi$, we shall define the jump operator $\llbracket \cdot \rrbracket$ by

$$
\llbracket \varphi \rrbracket:=\left.\left(\left.\varphi\right|_{K}\right)\right|_{\sigma}-\left.\left(\left.\varphi\right|_{L}\right)\right|_{\sigma} .
$$

We set $\llbracket \varphi \rrbracket_{\sigma}:=\left.\varphi\right|_{\sigma}$ for any $\sigma \in \mathcal{E}_{h}^{\text {ext }}$. We next associate with each $K \in \mathcal{T}_{h}$ and each $\sigma \in \mathcal{E}_{K}$ a 
weight $\omega_{K, \sigma}$ such that

$$
\begin{aligned}
0 \leq \omega_{K, \sigma} \leq 1 & \forall K \in \mathcal{T}_{h}, \forall \sigma \in \mathcal{E}_{K}, \\
\omega_{K, \sigma}+\omega_{L, \sigma}=1 & \forall \sigma=\sigma_{K, L} \in \mathcal{E}_{h}^{\text {int }}, \\
\omega_{K, \sigma}=1 & \forall \sigma \in \mathcal{E}_{h}^{\text {ext }} \text { and } K \in \mathcal{T}_{h} \text { such that } \sigma \in \mathcal{E}_{K} .
\end{aligned}
$$

For $\sigma=\sigma_{K, L} \in \mathcal{E}_{h}^{\text {int }}$, we define the weighted average operator $\left\{[\cdot\}_{\omega}\right.$ by

$$
\left\{\{\varphi\}_{\omega}:=\left.\omega_{K, \sigma}\left(\left.\varphi\right|_{K}\right)\right|_{\sigma}+\left.\omega_{L, \sigma}\left(\left.\varphi\right|_{L}\right)\right|_{\sigma}\right.
$$

whereas for $\sigma \in \mathcal{E}_{h}^{\text {ext }},\left\{\{\varphi\}_{\omega}:=\left.\varphi\right|_{\sigma}\right.$. Recall that we have denoted by $a$ the diffusion coefficient. Two basic choices for the weights in $\{a a\}_{\omega} \omega$ on a side $\sigma=\sigma_{K, L} \in \mathcal{E}_{h}^{\text {int }}$ are:

$$
\omega_{K, \sigma}=\omega_{L, \sigma}=\frac{1}{2}
$$

which corresponds to the arithmetic averaging, and

$$
\omega_{K, \sigma}=\frac{a_{L}}{a_{K}+a_{L}}, \quad \omega_{L, \sigma}=\frac{a_{K}}{a_{K}+a_{L}},
$$

which corresponds to the harmonic averaging. The present paper is done as generally as possible, for different numerical methods and different estimates using different types of the averages introduced above. We do not necessarily promote the harmonic averaging (2.5); we only show below that it can lead, in contrast to the arithmetic average (2.4), to robust a posteriori error estimates for the error measured in the energy norm. Finally, we denote by $\{4 \varphi\}$ the standard average operator with $\omega_{K, \sigma}=\omega_{L, \sigma}=\frac{1}{2}$ and $\{\{\varphi\}\}:=\left.\varphi\right|_{\sigma}$ for $\sigma \in \mathcal{E}_{h}^{\text {ext }}$. We use the same type of notation also for the meshes $\mathcal{D}_{h}$ and $\mathcal{S}_{h}$.

We shall be working below with numerical methods whose approximate solution can be represented by continuous piecewise affine functions on the primal simplicial mesh $\mathcal{T}_{h}$, with value 0 on the boundary of $\Omega$. We denote this space by $X_{h}^{0}$. The basis of $X_{h}^{0}$ is spanned by the pyramidal ("hat") functions $\psi_{V}$ associated with the vertices $V \in \mathcal{V}_{h}^{\text {int }}$, such that $\psi_{V}(U)=\delta_{V U}, U \in \mathcal{V}_{h}$, $\delta$ being the Kronecker delta.

Let $S \subset \Omega$. We denote by $(\cdot, \cdot)_{S}$ the $L^{2}$-scalar product on $S$ and by $\|\cdot\|_{S}$ the associated norm; when $S=\Omega$, the index dropped off. We mean by $|S|$ the Lebesgue measure of $S$, by $|\sigma|$ the $(d-1)$-dimensional Lebesgue measure of $\sigma \subset \mathbb{R}^{d-1}$, and in particular by $|\mathbf{s}|$ the length of a segment s. Next, $H^{1}(S)$ is the Sobolev space of functions with square-integrable weak derivatives and $H_{0}^{1}(S)$ is its subspace of functions with traces vanishing on $\partial S$. Finally, $\mathbf{H}(\operatorname{div}, S)$ is the space of functions with square-integrable weak divergences, $\mathbf{H}(\operatorname{div}, S)=\left\{\mathbf{v} \in \mathbf{L}^{2}(S) ; \nabla \cdot \mathbf{v} \in L^{2}(S)\right\}$, and $\langle\cdot, \cdot\rangle_{\partial S}$ stands for the appropriate duality pairing on $\partial S$.

\subsection{Assumptions}

We shall suppose that $f(\mathbf{x}) \in L^{2}(\Omega)$ and that $a(\mathbf{x})$ is a piecewise constant scalar-valued function. We in particular consider cases where $a$ is piecewise constant on the triangulation $\mathcal{T}_{h}$ and cases where $a$ is piecewise constant on the dual partition $\mathcal{D}_{h}$. This assumption corresponds to a situation where there exist subdomains of the domain $\Omega$ where $a$ is constant and where the meshes $\left(\mathcal{T}_{h}\right.$ or $\mathcal{D}_{h}$ ) are conforming with respect to these subdomains. We denote by $c_{a, K}$ and $C_{a, K}$ for all $K \in \mathcal{T}_{h}$ the best positive constants such that $c_{a, K} \leq a(\mathbf{x}) \leq C_{a, K}$ for all $\mathbf{x} \in K$. Similar notation will be used also for $D \in \mathcal{D}_{h}$, for $\mathcal{T}_{K}, K \in \mathcal{T}_{h}$, or for the entire domain. 


\subsection{Poincaré and Friedrichs inequalities}

Let $D$ be a polygon or a polyhedron. The Poincaré inequality states that

$$
\left\|\varphi-\varphi_{D}\right\|_{D}^{2} \leq C_{\mathrm{P}, D} h_{D}^{2}\|\nabla \varphi\|_{D}^{2} \quad \forall \varphi \in H^{1}(D),
$$

where $\varphi_{D}$ is the mean of the function $\varphi$ over $D$ given by $\varphi_{D}:=(\varphi, 1)_{D} /|D|$ and where the constant $C_{\mathrm{P}, D}$ can for each convex $D$ be evaluated as $1 / \pi^{2}$, cf. $[42,11]$. To evaluate $C_{\mathrm{P}, D}$ for nonconvex elements $D$ is more complicated but it still can be done, cf. [29, Lemma 10.2] or [18, Section 2].

Let the polygon/polyhedron $D$ be such that $|\partial \Omega \cap \partial D| \neq 0$. Then the Friedrichs inequality states that

$$
\|\varphi\|_{D}^{2} \leq C_{\mathrm{F}, D, \partial \Omega} h_{D}^{2}\|\nabla \varphi\|_{D}^{2} \quad \forall \varphi \in H^{1}(D) \text { such that } \varphi=0 \text { on } \partial \Omega \cap \partial D .
$$

As long as $D$ and $\partial \Omega$ are such that there exists a vector $\mathbf{b} \in \mathbb{R}^{d}$ such that for almost all $\mathbf{x} \in D$, the first intersection of $\mathcal{B}_{\mathbf{x}}$ and $\partial D$ lies in $\partial \Omega$, where $\mathcal{B}_{\mathbf{x}}$ is the straight semi-line defined by the origin $\mathbf{x}$ and the vector $\mathbf{b}$, the constant $C_{\mathrm{F}, D, \partial \Omega}$ can be taken equal to 1 , cf. [56, Remark 5.8]. To evaluate $C_{\mathrm{F}, D, \partial \Omega}$ in the general case is more complicated but it still can be done, cf. [56, Remark 5.9] or [18, Section 3].

\subsection{Continuous problem}

We define a bilinear form $\mathcal{B}$ by

$$
\mathcal{B}(p, q):=(a \nabla p, \nabla q) \quad p, q \in H_{0}^{1}(\Omega) .
$$

The weak formulation of problem (1.1a) $-(1.1 \mathrm{~b})$ is to find $p \in H_{0}^{1}(\Omega)$ such that

$$
\mathcal{B}(p, q)=(f, q) \quad \forall q \in H_{0}^{1}(\Omega) .
$$

The energy norm of problem (1.1a)-(1.1b) is defined by

$$
\|q\|\left\|^{2}:=\mathcal{B}(q, q)=\right\| a^{\frac{1}{2}} \nabla q \|^{2}, \quad q \in H_{0}^{1}(\Omega) .
$$

We will present our a posteriori error estimates in this norm. Following the approaches of Angermann [4] or Verfürth [55] and Chaillou and Suri [19, 20], we will also present a posteriori error estimates in a dual norm. We will use the $H^{-1}$ norm of the residual given by

$$
\|q\|_{\#}:=\sup _{\varphi \in H_{0}^{1}(\Omega)} \frac{\mathcal{B}(q, \varphi)}{\|\nabla \varphi\|}, \quad q \in H_{0}^{1}(\Omega)
$$

for this purpose.

Remark 2.1 (Energy and dual norms). The energy norm (2.10) and the dual norm (2.11) coincide when $a=1$. They, however, differ in general. The energy norm (2.10) admits a local decomposition and is easily computable. The dual norm (2.11) is a global norm and its practical computation is not obvious except of particular cases. In any case, however, it is immediate from (2.11) that there exist easily and locally computable upper and lower bounds for $\|\cdot \cdot\|_{\#}$ :

$$
\frac{\left\|a^{\frac{1}{2}} \nabla q\right\|^{2}}{\|\nabla q\|} \leq\|q\|_{\#} \leq\|a \nabla q\| \quad \forall q \in H_{0}^{1}(\Omega) .
$$




\section{Some $H^{1}$-conforming methods and their mutual relations}

The purpose of this section is to recall several classical numerical methods for problem (1.1a)-(1.1b) and their mutual relations. This section can be skipped and read later; the results of Sections 4 and 5 below are completely independent of it.

\subsection{Definitions}

We start by giving the definitions.

Definition 3.1 (Weighted cell-centered finite volume method). Let $\mathcal{D}_{h}$ be the Voronoï grid given by the vertices from $\mathcal{V}_{h}$, cf. Eymard et al. [29] (this requires that the vertices $V \in \mathcal{V}_{h}^{\text {ext }}$ are suitably placed so that $\bar{\Omega}=\bigcup_{D \in \mathcal{D}_{h}} D$ ). Let next $\mathcal{N}(D)$ denote the set of "neighbors" of $D \in \mathcal{D}_{h}$, i.e., of such $E \in \mathcal{D}_{h}$ that $\sigma_{D, E}:=\partial D \cap \partial E$ is such that $\left|\sigma_{D, E}\right| \neq 0$; in such a case, let $d_{D, E}$ stand for the Euclidean distance of the associated vertices $V_{D}$ and $V_{E}$. Let finally the diffusion coefficient $a$ be piecewise constant on $\mathcal{D}_{h}$. Then the weighted cell-centered finite volume method for problem (1.1a)-(1.1b) reads: find the values $p_{D}, D \in \mathcal{D}_{h}^{\text {int }}$, with $p_{D}=0$ for all $D \in \mathcal{D}_{h}^{\text {ext }}$, such that

$$
\left.-\sum_{E \in \mathcal{N}(D)}\{a\}\right\}_{\omega} \frac{\left|\sigma_{D, E}\right|}{d_{D, E}}\left(p_{E}-p_{D}\right)=(f, 1)_{D} \quad \forall D \in \mathcal{D}_{h}^{\text {int }} .
$$

Recall the notation $\psi_{V_{D}}$ for the hat function associated with the vertex $V_{D}$ associated with the dual volume $D \in \mathcal{D}_{h}$. Then the solution of (3.1) can be interpreted as a function $p_{h} \in X_{h}^{0}, p_{h}=$ $\sum_{D \in \mathcal{D}_{h}} p_{D} \psi_{V_{D}}$. The two basic choices for the weights in $\{\{a\}\} \omega$ are the arithmetic averaging (2.4) and the harmonic averaging (2.5).

Definition 3.2 (Vertex-centered finite volume method). Let the dual grid $\mathcal{D}_{h}$, as described in Section 2.1, consist of polygonal/polyhedral dual volumes and let $a$ be piecewise constant on $\mathcal{T}_{h}$ so that $a$ is not double-valued on $\mathcal{F}_{h}^{\text {int }}$, the interior sides of $\mathcal{D}_{h}$. Then the vertex-centered finite volume method for problem (1.1a)-(1.1b) reads: find $p_{h} \in X_{h}^{0}$ such that

$$
-\left\langle a \nabla p_{h} \cdot \mathbf{n}_{D}, 1\right\rangle_{\partial D}=(f, 1)_{D} \quad \forall D \in \mathcal{D}_{h}^{\text {int }} .
$$

Definition 3.3 (Weighted vertex-centered finite volume method). Let the dual grid $\mathcal{D}_{h}$, as described in Section 2.1, consist of polygonal/polyhedral dual volumes. Then we can design a weighted vertex-centered finite volume method for problem (1.1a)-(1.1b) as follows: find $p_{h} \in X_{h}^{0}$ such that

$$
-\left\langle\{\{a\}\}_{\omega} \nabla p_{h} \cdot \mathbf{n}_{D}, 1\right\rangle_{\partial D}=(f, 1)_{D} \quad \forall D \in \mathcal{D}_{h}^{\text {int }} .
$$

Remark 3.4 (Arithmetic/harmonic averaging in the vertex-centered finite volume method). We first remark that when $a$ is piecewise constant on $\mathcal{T}_{h}$, the above Definition 3.3 coincides with the standard Definition 3.2, which is known to lead to arithmetic-like averaging of $a$. When, however, $a$ is piecewise constant on $\mathcal{D}_{h}$, then as in the cell-centered finite volume case of Definition 3.1, the two basic choices for the weights $\{\{a\}\}_{\omega}$ in (3.3) are (2.4) and (2.5), leading respectively to arithmetic and harmonic averaging of $a$.

Definition 3.5 (Finite element method). The finite element method for problem (1.1a)-(1.1b) reads: find $p_{h} \in X_{h}^{0}$ such that

$$
\left(a \nabla p_{h}, \nabla \psi_{V}\right)_{\mathcal{T}_{V}}=\left(f, \psi_{V}\right)_{\mathcal{T}_{V}} \quad \forall V \in \mathcal{V}_{h}^{\text {int }}
$$


Recall that in the above definition, $\psi_{V}$ is the hat function associated with the vertex $V$ and $\mathcal{T}_{V}$ is the set of elements of the mesh $\mathcal{T}_{h}$ which share the vertex $V$.

Definition 3.6 (Finite element method with harmonic averaging). Let the dual grid $\mathcal{D}_{h}$, as described in Section 2.1, consist of polygonal/polyhedral dual volumes and let $a$ be piecewise constant on $\mathcal{D}_{h}$. Let us define $\tilde{a}$ by

$$
\left.\tilde{a}\right|_{K}=\left(\frac{\left(a^{-1}, 1\right)_{K}}{|K|}\right)^{-1} \quad \forall K \in \mathcal{T}_{h} .
$$

Then we can define a finite element method with harmonic averaging for problem (1.1a)-(1.1b) as: find $p_{h} \in X_{h}^{0}$ such that

$$
\left(\tilde{a} \nabla p_{h}, \nabla \psi_{V}\right)_{\mathcal{T}_{V}}=\left(f, \psi_{V}\right)_{\mathcal{T}_{V}} \quad \forall V \in \mathcal{V}_{h}^{\text {int }} .
$$

Remark 3.7 (Arithmetic/harmonic averaging in the finite element method). We remark that the difference between the matrices of (3.4) and (3.6) corresponds to the difference between the matrices of the piecewise affine nonconforming finite element method and that of the hybridization of the lowest-order Raviart-Thomas-Nédélec mixed finite element method in that the first ones use the arithmetic and the second ones use the harmonic averaging of the diffusion coefficient $a$, cf. [6] or [30, Lemma 8.1]. In particular, by Definitions 3.5 and 3.6, one has in the finite element method the choice between the arithmetic and the harmonic averaging as in the finite volume ones.

\subsection{Equivalences}

We are now ready to recall several equivalence results between the above methods.

Lemma 3.8 (Equivalence between matrices of finite elements and vertex-centered finite volumes). Let $D \in \mathcal{D}_{h}$, as described in Section 2.1, have Lipschitz-continuous boundaries and let $|\sigma \cap D|=$ $|\sigma| / d$ for each side $\sigma \in \mathcal{E}_{h}^{\text {int }}$ with the vertex $V_{D} \in \mathcal{V}_{h}^{\text {int }}$ and the associated dual volume $D \in \mathcal{D}_{h}^{\text {int }}$. Let, moreover, the diffusion coefficient a be piecewise constant on $\mathcal{T}_{h}$. Then, for all $p_{h} \in X_{h}^{0}$,

$$
\left(a \nabla p_{h}, \nabla \psi_{V_{D}}\right)_{\mathcal{T}_{V_{D}}}=-\left\langle a \nabla p_{h} \cdot \mathbf{n}_{D}, 1\right\rangle_{\partial D} \quad \forall D \in \mathcal{D}_{h}^{\text {int }} .
$$

Proof. Employing the Green theorem and the finite elements basis functions form, see [9, Lemma 3] for $d=2$.

Lemma 3.9 (Equivalence between matrices of finite elements and cell-centered finite volumes). Let $d=2$, let $\mathcal{T}_{h}$ be Delaunay, that is let the circumcircle of each triangle does not contain any vertex in its interior, and let, moreover, no circumcenters of boundary triangles lie outside the domain $\Omega$. Let $\mathcal{D}_{h}$ be the Voronoï grid given by the vertices from $\mathcal{V}_{h}$ and let $a=1$. Then, for all $p_{h} \in X_{h}^{0}$,

$$
\left(\nabla p_{h}, \nabla \psi_{V_{D}}\right)_{\mathcal{T}_{V_{D}}}=-\sum_{E \in \mathcal{N}(D)} \frac{\left|\sigma_{D, E}\right|}{d_{D, E}}\left(p_{E}-p_{D}\right) \quad \forall D \in \mathcal{D}_{h}^{\text {int }} .
$$

Proof. See [29, Section III.12].

Remark 3.10 (Relation between finite elements and cell-centered finite volumes if $d=3$ ). We remark that the above lemma does not generalize to three space dimensions, see, e.g., Letniowski [38] or Putti and Cordes [45]. 
Lemma 3.11 (Equivalence between right-hand sides of finite elements and finite volumes). Let $\mathcal{D}_{h}$ be as described in Section 2.1 and let $|D \cap K|=|K| /(d+1)$ for each dual volume $D \in \mathcal{D}_{h}^{\text {int }}$ and each element $K \in \mathcal{T}_{V_{D}}$. Let, moreover, $f$ be piecewise constant on $\mathcal{T}_{h}$. Then

$$
\left(f, \psi_{V_{D}}\right)_{\mathcal{T}_{V_{D}}}=(f, 1)_{D} \quad \forall D \in \mathcal{D}_{h}^{\text {int }} .
$$

Proof. Straightforward using the condition $|D \cap K|=|K| /(d+1)$ for $D \in \mathcal{D}_{h}^{\text {int }}$ and $K \in \mathcal{T}_{V_{D}}$ and a quadrature formula for affine functions on simplices.

\subsection{Consequences}

The following corollaries are obvious consequences of the previous lemmas.

Corollary 3.12 (Equivalence between finite elements and vertex-centered finite volumes). Let the assumptions of Lemmas 3.8 and 3.11 be verified. Then the finite element method given by Definition 3.5 and the vertex-centered finite volume methods given by Definitions 3.2 and 3.3 produce the same discrete problems/linear systems.

Corollary 3.13 (Local conservativity of the finite element method on dual grids). Let the assumptions of Lemmas 3.8 and 3.11 be verified. Then the finite element method given by Definition 3.5 is locally conservative over the dual grid $\mathcal{D}_{h}^{\text {int }}$.

Corollary 3.14 (Equivalence between weighted cell- and vertex-centered finite volumes). Let $d=2$, let $\mathcal{T}_{h}$ be Delaunay, let no circumcenters of boundary triangles lie outside the domain $\Omega$, and let $\mathcal{D}_{h}$ be the Voronoï grid given by the vertices from $\mathcal{V}_{h}$. Let next a be piecewise constant on $\mathcal{D}_{h}$. Then the weighted cell-centered finite volume method given by Definition 3.1 and the weighted vertex-centered finite volume method given by Definition 3.3 produce the same discrete problems/linear systems.

\subsection{Remarks}

We finish this section by some additional remarks.

Remark 3.15 (Local conservativity of the finite element method). Corollary 3.13 should be understood in the following sense: First of all, equation (3.2) states that the sum of fluxes entering/leaving the given dual volume $D \in \mathcal{D}_{h}^{\text {int }}$ equals the sources on this element. Secondly, rewriting $-\left\langle a \nabla p_{h} \cdot \mathbf{n}_{D}, 1\right\rangle_{\partial D}$ as $-\sum_{E \in \mathcal{N}(D)}\left\langle a \nabla p_{h} \cdot \mathbf{n}_{D}, 1\right\rangle_{\sigma_{D, E}}$ and noticing that the quantity $a \nabla p_{h} \cdot \mathbf{n}_{D}$ is single-valued on the side $\sigma_{D, E}$ under the given assumptions, local mass balance, in the sense that the mass leaving from one element $(D)$ enters its neighbor $(E)$, is likewise satisfied. Consequently, the finite element method is well locally mass conservative on the dual mesh $\mathcal{D}_{h}^{\text {int }}$, even if it is not locally mass conservative on the primal simplicial mesh $\mathcal{T}_{h}$. Remark finally that the above assertions are only valid exactly if $a$ and $f$ are piecewise constant on $\mathcal{T}_{h}$. For general coefficients $a$ and $f$, the local mass conservativity on $\mathcal{D}_{h}^{\text {int }}$ only holds up to a numerical quadrature/data oscillation.

Remark 3.16 (Choice of the dual grids). In the above developments, a large freedom is left in what concerns the actual choice of the dual grids $\mathcal{D}_{h}$. The basic and most frequently used grid satisfying both the assumptions of Lemmas 3.8 and 3.11 is given by straight lines connecting the triangle barycentres through the midpoints of the edges of $\mathcal{T}_{h}$ if $d=2$, see Figure 1, and similarly if $d=3$.

Remark 3.17 (Finite difference method). Let $\mathcal{D}_{h}$ consist of squares if $d=2$ and cubes if $d=3$. Then the finite difference method for problem (1.1a)-(1.1b) coincides with cell-centered finite volume one given by Definition 3.1, cf. Eymard et al. [29]. 
Remark 3.18 (Tensor-valued diffusion coefficients). In problem (1.1a)-(1.1b), we could also consider a tensor-valued diffusion coefficient $\mathbf{A}$ in place of the scalar-valued diffusion coefficient $a$. Definitions 3.5 and 3.6 would in this case contain $\mathbf{A}$ in place of $a$ and similarly for Definitions 3.2 and 3.3. Then, for $\mathbf{A}$ piecewise constant on $\mathcal{T}_{h}$, Lemma 3.8 still holds true and similarly for Corollaries 3.12 and 3.13 .

\section{Guaranteed a posteriori error estimates}

We present our main upper bound results in this section. We first give an abstract framework related to the Prager-Synge theorem, then state and prove our a posteriori error estimates, discuss several different ways of construction of an equilibrated flux, and finally give a series of remarks. Note that the results of this section are presented generally, without a notion of any numerical scheme. For our a posteriori error estimates of Theorem 4.4 or Corollary 4.6, we only need Assumption 4.4. The different constructions of the equilibrated flux $\mathbf{t}_{h}$ are then presented under Assumption 4.9 solely.

\subsection{A simple abstract framework and its relation to the Prager-Synge theorem}

We present here a simple abstract a posteriori error estimate for problem (1.1a)-(1.1b). The basic ideas can be traced back to the Prager-Synge equality [44], the hypercircle method, cf. Synge [51], Ladevèze [36], Haslinger and Hlaváček [33], Vacek [52], Hlaváček et al. [34], or Repin [46].

Theorem 4.1 (Abstract energy norm a posteriori error estimate). Let $p$ be the weak solution of problem (1.1a)-(1.1b) and let $p_{h} \in H_{0}^{1}(\Omega)$ be arbitrary. Then

$$
\left\|\left|p-p_{h}\right|\right\|=\inf _{\mathbf{t} \in \mathbf{H}(\operatorname{div}, \Omega)} \sup _{\varphi \in H_{0}^{1}(\Omega),\|\varphi\| \|=1}\left\{|(f-\nabla \cdot \mathbf{t}, \varphi)|+\left|\left(a \nabla p_{h}+\mathbf{t}, \nabla \varphi\right)\right|\right\} .
$$

Proof. We first notice that

$$
\left\|\left|p-p_{h} \|\right|=\mathcal{B}\left(p-p_{h}, \frac{p-p_{h}}{\left\|p-p_{h} \mid\right\|}\right)\right.
$$

by (2.10). Clearly, as $\varphi:=\left(p-p_{h}\right) /\left\|p-p_{h}\right\| \mid \in H_{0}^{1}(\Omega)$, we immediately have $\mathcal{B}(p, \varphi)=(f, \varphi)$ by (2.9). Using this we obtain, for an arbitrary $\mathbf{t} \in \mathbf{H}(\operatorname{div}, \Omega)$ and employing (2.8) and the Green theorem,

$$
\begin{aligned}
\mathcal{B}\left(p-p_{h}, \varphi\right) & =(f, \varphi)-\left(a \nabla p_{h}, \nabla \varphi\right)=(f, \varphi)-\left(a \nabla p_{h}+\mathbf{t}, \nabla \varphi\right)+(\mathbf{t}, \nabla \varphi) \\
& \leq|(f-\nabla \cdot \mathbf{t}, \varphi)|+\left|\left(a \nabla p_{h}+\mathbf{t}, \nabla \varphi\right)\right| .
\end{aligned}
$$

From here, it is enough to note that $\|\varphi \varphi\|=1$ and that $\mathbf{t} \in \mathbf{H}(\operatorname{div}, \Omega)$ was chosen arbitrary to conclude that the right-hand side term of (4.1) is an upper bound on the left-hand side one. For the converse estimate, it suffices to set $\mathbf{t}=-a \nabla p$ and to use (2.9), the Cauchy-Schwarz inequality, and the fact that $\|\varphi\| \|=1$.

Similar arguments lead to the following corollary:

Corollary 4.2 (Abstract dual norm a posteriori error estimate). Let the assumptions of Theorem 4.1 be verified. Then

$$
\left\|\left|p-p_{h}\right|\right\|_{\#}=\inf _{\mathbf{t} \in \mathbf{H}(\operatorname{div}, \Omega)} \sup _{\varphi \in H_{0}^{1}(\Omega),\|\nabla \varphi\|=1}\left\{|(f-\nabla \cdot \mathbf{t}, \varphi)|+\left|\left(a \nabla p_{h}+\mathbf{t}, \nabla \varphi\right)\right|\right\} .
$$


Remark 4.3 (Relation to the Prager-Synge equality). The Prager-Synge equality [44] states, with the assumptions of Theorem 4.1, that

$$
\left\|p-p_{h} \mid\right\|^{2}+\left\|a^{\frac{1}{2}} \nabla p+a^{-\frac{1}{2}} \mathbf{t}\right\|^{2}=\left\|a^{\frac{1}{2}} \nabla p_{h}+a^{-\frac{1}{2}} \mathbf{t}\right\|^{2}
$$

for any $\mathbf{t} \in \mathbf{H}(\operatorname{div}, \Omega)$ such that $\nabla \cdot \mathbf{t}=f$. This result leads to

$$
\left\|p-p_{h}\right\| \mid \leq \inf _{\mathbf{t} \in \mathbf{H}(\operatorname{div}, \Omega) ; \nabla \cdot \mathbf{t}=f}\left\|a^{\frac{1}{2}} \nabla p_{h}+a^{-\frac{1}{2}} \mathbf{t}\right\|,
$$

which is similar to (4.1). The important difference, however, is that the minimization set is here restrained to such $\mathbf{t} \in \mathbf{H}(\operatorname{div}, \Omega)$ that satisfy $\nabla \cdot \mathbf{t}=f$, which is a rather restrictive condition, whereas in (4.1), the minimization set is unconstrained.

\subsection{A posteriori error estimate}

Starting from Theorem 4.1, we now give a fully computable a posteriori error estimate. Essential is Assumption 4.4 below which enables to easily estimate the first term on the right-hand side of (4.1), related to a negative norm. Remark that this assumption is very general and does not need neither the definition of a particular numerical method, nor any specific form of the equilibrated flux $\mathbf{t}_{h}$. Examples of the construction of convenient fluxes $\mathbf{t}_{h}$ are given in Section 4.3 below.

In order to present the result of this section as generally as possible, let us suppose that the domain $\Omega$ is partitioned by a mesh $\mathcal{D}_{h}^{*}$, consisting in general of elements in the interior of the domain, $\mathcal{D}_{h}^{\text {int,* }}$, and of elements near the boundary, $\mathcal{D}_{h}^{\text {ext,* }}\left(|\partial \Omega \cap \partial D| \neq 0\right.$ for all $\left.D \in \mathcal{D}_{h}^{\text {ext }, *}\right)$. The meshes $\mathcal{D}_{h}^{*}$ will differ in different types of construction of the equilibrated flux $\mathbf{t}_{h}$. Three main possibilities exist. Either $\mathcal{D}_{h}^{*}$ is given by the dual mesh $\mathcal{D}_{h}$ of Section 2.1 , i.e., $\mathcal{D}_{h}^{\text {int,* }}=\mathcal{D}_{h}^{\text {int }}$ and $\mathcal{D}_{h}^{\text {ext,*}}=\mathcal{D}_{h}^{\text {ext }}$; or $\mathcal{D}_{h}^{\text {int,* }}=\mathcal{S}_{h}$ and $\mathcal{D}_{h}^{\text {ext,*}}=\emptyset$, where $\mathcal{S}_{h}$ is given in Section 2.1 ; or $\mathcal{D}_{h}^{\text {int,* }}=\mathcal{T}_{h}$ and $\mathcal{D}_{h}^{\text {ext, }, *}=\emptyset$.

Let us now make the essential assumption:

Assumption 4.4 (Equilibrated flux). Suppose that there is a flux $\mathbf{t}_{h} \in \mathbf{H}(\operatorname{div}, \Omega)$, arbitrary but such that

$$
\left(\nabla \cdot \mathbf{t}_{h}, 1\right)_{D}=(f, 1)_{D} \quad \forall D \in \mathcal{D}_{h}^{\text {int,* }} .
$$

With these notations and assumptions, we can now state our main results:

Theorem 4.5 (A guaranteed energy norm a posteriori error estimate). Let $p$ be the weak solution of problem (1.1a)-(1.1b) and let $p_{h} \in H_{0}^{1}(\Omega)$ be arbitrary. Let Assumption 4.4 hold. Then

$$
\left\|\left|p-p_{h} \|\right| \leq\left\{\sum_{D \in \mathcal{D}_{h}^{*}}\left(\eta_{\mathrm{R}, D}+\eta_{\mathrm{DF}, D}\right)^{2}\right\}^{\frac{1}{2}},\right.
$$

where the diffusive flux estimator $\eta_{\mathrm{DF}, D}$ is given by

$$
\eta_{\mathrm{DF}, D}:=\left\|a^{\frac{1}{2}} \nabla p_{h}+a^{-\frac{1}{2}} \mathbf{t}_{h}\right\|_{D} \quad D \in \mathcal{D}_{h}^{*},
$$

and the residual estimator $\eta_{\mathrm{R}, D}$ is given by

$$
\eta_{\mathrm{R}, D}:=m_{D, a}\left\|f-\nabla \cdot \mathbf{t}_{h}\right\|_{D} \quad D \in \mathcal{D}_{h}^{*},
$$

where

$$
m_{D, a}^{2}:=C_{\mathrm{P}, D} \frac{h_{D}^{2}}{c_{a, D}} \quad D \in \mathcal{D}_{h}^{\mathrm{int}, *}, \quad m_{D, a}^{2}:=C_{\mathrm{F}, D, \partial \Omega} \frac{h_{D}^{2}}{c_{a, D}} \quad D \in \mathcal{D}_{h}^{\text {ext,* }},
$$

with $C_{\mathrm{P}, D}$ the constant from the Poincaré inequality (2.6) and $C_{\mathrm{F}, D, \partial \Omega}$ the constant from the Friedrichs inequality (2.7). 
Proof. Set $\mathbf{t}=\mathbf{t}_{h}$ in Theorem 4.1. Note that, for each $D \in \mathcal{D}_{h}^{\text {int,*, }}$

$$
\left|\left(f-\nabla \cdot \mathbf{t}_{h}, \varphi\right)_{D}\right|=\left|\left(f-\nabla \cdot \mathbf{t}_{h}, \varphi-\varphi_{D}\right)_{D}\right| \leq\left.\eta_{\mathrm{R}, D}|||\varphi|\right|_{D},
$$

using Assumption 4.4, the Poincaré inequality (2.6), the Cauchy-Schwarz inequality, and the definition (2.10) of the energy norm. We cannot use a similar approach also for $D \in \mathcal{D}_{h}^{\text {ext,* }}$ since there is no local conservativity assumed on these volumes (recall that Assumption 4.4 is only supposed to hold for $\left.D \in \mathcal{D}_{h}^{\text {int,* }}\right)$. On the other hand, however, $\varphi=0$ on $\partial D \cap \partial \Omega$, whence

$$
\left|\left(f-\nabla \cdot \mathbf{t}_{h}, \varphi\right)_{D}\right| \leq \eta_{\mathrm{R}, D}|| \varphi \mid \|_{D}
$$

for each $D \in \mathcal{D}_{h}^{\text {ext,* }}$, using the Friedrichs inequality (2.7), the Cauchy-Schwarz inequality, and the definition (2.10) of the energy norm. Finally, $\left|\left(a \nabla p_{h}+\mathbf{t}, \nabla \varphi\right)_{D}\right| \leq \eta_{\mathrm{DF}, D} \mid\|\varphi\|_{D}$ is immediate using the fact that $a$ is positive and scalar and the Cauchy-Schwarz inequality. Hence it now suffices to use the Cauchy-Schwarz inequality and to notice that $\|\varphi \varphi\|=1$ in order to conclude the proof.

The proof of the following corollary is completely similar:

Corollary 4.6 (A guaranteed dual norm a posteriori error estimate). Let the assumptions of Theorem 4.5 be verified. Then

$$
\left\|p-p_{h}\right\|_{\#} \leq\left\{\sum_{D \in \mathcal{D}_{h}^{*}}\left(\eta_{\mathrm{R}, D}+\eta_{\mathrm{DF}, D}\right)^{2}\right\}^{\frac{1}{2}},
$$

with the diffusive flux estimator $\eta_{\mathrm{DF}, D}$ given by

$$
\eta_{\mathrm{DF}, D}:=\left\|a \nabla p_{h}+\mathbf{t}_{h}\right\|_{D} \quad D \in \mathcal{D}_{h}^{*}
$$

and the residual estimator $\eta_{\mathrm{R}, D}$ given by

$$
\eta_{\mathrm{R}, D}:=m_{D}\left\|f-\nabla \cdot \mathbf{t}_{h}\right\|_{D} \quad D \in \mathcal{D}_{h}^{*},
$$

where

$$
m_{D}^{2}:=C_{\mathrm{P}, D} h_{D}^{2} \quad D \in \mathcal{D}_{h}^{\text {int,* }}, \quad m_{D}^{2}:=C_{\mathrm{F}, D, \partial \Omega} h_{D}^{2} \quad D \in \mathcal{D}_{h}^{\text {ext,* }} .
$$

Remark 4.7 (Assumptions of Theorem 4.5 and Corollary 4.6). Note that for Theorem 4.5 and Corollary 4.6, no additional assumptions like a polynomial form of the data $a$ or $f$, a polynomial form of the approximate solution $p_{h}$, or a shape regularity of the mesh $\mathcal{D}_{h}^{*}$ are needed.

Remark 4.8 (Comparison of the estimators of Theorem 4.5 and of Corollary 4.6). The estimators of Theorem 4.5 and of Corollary 4.6 coincide when $a=1$. We find the estimators of Corollary 4.6 more physical as they measure the misfit between the true fluxes $a \nabla p_{h}$ and $\mathbf{t}_{h}$ and not their energy counterparts $a^{\frac{1}{2}} \nabla p_{h}$ and $a^{-\frac{1}{2}} \mathbf{t}_{h}$ in $\eta_{\mathrm{DF}, D}$. The estimators of Corollary 4.6 also do not involve the constant $c_{a, D}$ in the residual estimators $\eta_{\mathrm{R}, D}$.

In order to use Theorem 4.5 and Corollary 4.6 in practice, we need to construct a (finitedimensional) equilibrated flux $\mathbf{t}_{h}$ satisfying Assumption 4.4. We will look for a suitable $\mathbf{t}_{h}$ in the lowest-order Raviart-Thomas-Nédélec space $\operatorname{RTN}\left(\mathcal{S}_{h}\right)$ defined over the fine simplicial mesh $\mathcal{S}_{h}$ of Section 2.1. The space $\mathbf{R T N}\left(\mathcal{S}_{h}\right)$ is a finite-dimensional subspace of $\mathbf{H}(\operatorname{div}, \Omega)$. It is a space of vector functions having on each $K \in \mathcal{S}_{h}$ the form $\left(a_{K}+d_{K} x, b_{K}+d_{K} y\right)^{t}$ if $d=2$ and $\left(a_{K}+d_{K} x, b_{K}+d_{K} y, c_{K}+d_{K} z\right)^{t}$ if $d=3$. Note that the requirement $\operatorname{RTN}\left(\mathcal{S}_{h}\right) \subset \mathbf{H}(\operatorname{div}, \Omega)$ imposes the continuity of the normal trace across all interior sides $\sigma$ of $\mathcal{S}_{h}, \sigma \in \mathcal{G}_{h}^{\text {int }}$, and recall that $\mathbf{v}_{h} \cdot \mathbf{n}_{\sigma}$ is a constant for all $\sigma \in \mathcal{G}_{h}$ for any function $\mathbf{v}_{h} \in \mathbf{R T N}\left(\mathcal{S}_{h}\right)$. The side fluxes $\left\langle\mathbf{v}_{h} \cdot \mathbf{n}_{\sigma}, 1\right\rangle_{\sigma}$ also represent the degrees of freedom of $\operatorname{RTN}\left(\mathcal{S}_{h}\right)$. For more details, we refer to $[16,49]$. RaviartThomas-Nédélec spaces have been used previously in a posteriori error estimation in a similar concept in $[46,24,39,35,15]$. 


\subsection{Constructions of the equilibrated flux $\mathrm{t}_{h}$}

We show here four different ways of constructing an equilibrated flux $\mathbf{t}_{h}$ satisfying Assumption 4.4. Let $\mathcal{D}_{h}$ be as defined in Section 2.1. In order to make the presentation general, independent of a particular numerical method, we will henceforth assume:

Assumption 4.9 (General local conservativity of the numerical scheme). Assume that $p_{h} \in X_{h}^{0}$ and that, for $\mathcal{D}_{h}$ defined in Section 2.1, there holds

$$
\left.-\left\langle\left\{a \nabla p_{h} \cdot \mathbf{n}_{D}\right\}\right\}_{\omega}, 1\right\rangle_{\partial D}=(f, 1)_{D} \quad \forall D \in \mathcal{D}_{h}^{\text {int }} .
$$

\subsubsection{Construction of $t_{h}$ by direct prescription}

We define $\mathbf{t}_{h} \in \mathbf{R T N}\left(\mathcal{S}_{h}\right)$ by

$$
\mathbf{t}_{h} \cdot \mathbf{n}_{\sigma}:=-\left\{\left\{a \nabla p_{h} \cdot \mathbf{n}_{\sigma}\right\}\right\}_{\omega} \quad \forall \sigma \in \mathcal{G}_{h},
$$

where the weights $\omega$ are the same as those in Assumption 4.9. Thus a simple (weighted) average of the normal components of the approximate flux $-a \nabla p_{h}$ over the sides of fine simplicial mesh $\mathcal{S}_{h}$ is used to define the equilibrated flux $\mathbf{t}_{h}$. Note that by this construction, $\left\langle\mathbf{t}_{h} \cdot \mathbf{n}_{D}, 1\right\rangle_{\partial D}=(f, 1)_{D}$ for all $D \in \mathcal{D}_{h}^{\text {int }}$ is immediate from Assumption 4.9, whence the validity of Assumption 4.4 follows by the Green theorem; we take here $\mathcal{D}_{h}^{*}=\mathcal{D}_{h}$, i.e., $\mathcal{D}_{h}^{\text {int,* }}=\mathcal{D}_{h}^{\text {int }}$ and $\mathcal{D}_{h}^{\text {ext,* }}=\mathcal{D}_{h}^{\text {ext }}$.

This construction, however, may suffer from two inconveniences. Firstly, whenever $D \in \mathcal{D}_{h}^{\text {int }}$ is nonconvex, the Poincaré constant $C_{\mathrm{P}, D}$ from (2.6) is no longer equal to $1 / \pi^{2}$ and its evaluation is much more difficult leading to less sharp estimates. The second inconvenience was pointed out in [21]: Assumption 4.4 in this case only holds on the dual volumes $D \in \mathcal{D}_{h}^{\text {int }}$, i.e., on patches $\mathcal{S}_{D}$ of simplices $K \in \mathcal{S}_{h}$, and not on each $K \in \mathcal{S}_{h}$. Consequently, the residual estimators $\eta_{\mathrm{R}, D}$ are not higher-order terms as in $[57,27]$ and may dominate the diffusive flux ones $\eta_{\mathrm{DF}, D}$. As a consequence, in multiple space dimensions, the effectivity index does not approach the optimal value of one (cf. the numerical experiments of Section 7.2.1 below). This conclusion does not hold true in one space dimension, see Section 7.1.1 below. The approaches of the three following sections improve on these two points (we present them in the energy norm setting, similar results in the dual norm setting are rather straightforward).

\subsubsection{Construction of $t_{h}$ by local minimization involving local linear systems solution}

The equilibrated flux $\mathbf{t}_{h}$ of this section should be used in Theorem 4.5 or Corollary 4.6 with $\mathcal{D}_{h}^{*}=\mathcal{D}_{h}$.

In [21], $\mathbf{t}_{h} \cdot \mathbf{n}_{\sigma}$ is given by (4.8) only on such sides $\sigma \in \mathcal{G}_{h}$ which are at the boundary of some dual volume $D \in \mathcal{D}_{h}^{\text {int }}$. By the Green theorem, this is sufficient for Assumption 4.4 to hold, with $\mathcal{D}_{h}^{\text {int,* }}=\mathcal{D}_{h}^{\text {int }}$. The remaining sides of the mesh $\mathcal{S}_{h}$ lie in the interior of some dual volume $D \in \mathcal{D}_{h}$ (or at the boundary of $\Omega$ ), so that $\mathbf{t}_{h} \cdot \mathbf{n}_{\sigma}$ can be chosen locally and independently by local minimization of $\eta_{\mathrm{R}, D}^{2}+\eta_{\mathrm{DF}, D}^{2}$ for each $D \in \mathcal{D}_{h}$, in function of these remaining degrees of freedom. This leads to a solution of a small linear system for each $D \in \mathcal{D}_{h}$ and helps the improve the effectivity index to a value close to one.

\subsubsection{Construction of $t_{h}$ by local minimization without local linear systems solution}

We suggest here an improvement of the previous approach which avoids the solution of any local linear system. In this section as well, we set $\mathcal{D}_{h}^{*}=\mathcal{D}_{h}$. 
The first step is to construct $\mathbf{t}_{1, h} \in \mathbf{R T N}\left(\mathcal{S}_{h}\right)$ given by (4.8). Let next a dual volume $D \in \mathcal{D}_{h}$ be fixed and let $\mathbf{t}_{1, D}$ be given by $\left.\mathbf{t}_{1, h}\right|_{D}$. In the second step, we then construct $\mathbf{t}_{2, D} \in \mathbf{R T N}\left(\mathcal{S}_{D}\right)$ given by (4.8) only for such sides $\sigma \in \mathcal{G}_{h}$ contained in $D$ which are at the boundary of some $E \in \mathcal{D}_{h}^{\text {int }}$. We require that $\mathbf{t}_{2, D}$ was such that $\left(\nabla \cdot \mathbf{t}_{2, D}, 1\right)_{K}=(f, 1)_{K}$ for all $K \in \mathcal{S}_{D}$, i.e., such that the local mass conservation is satisfied for every element $K$ of the fine simplicial submesh $\mathcal{S}_{D}$ of the dual volume $D$ and not only for the dual volume $D$. Let $D \in \mathcal{D}_{h}^{\text {int }}$. The crucial point is that it follows from the fact that we have the local conservation on $D \in \mathcal{D}_{h}^{\text {int }},\left(\nabla \cdot \mathbf{t}_{2, D}, 1\right)_{D}=\left\langle\mathbf{t}_{2, D} \cdot \mathbf{n}_{D}, 1\right\rangle_{\partial D}=(f, 1)_{D}$, this can be done without any (local) linear system solution by choosing the flux over one interior side and a sequential construction (the mass balance on the last element $K \in \mathcal{S}_{D}$ that we come to will be satisfied as $\left.\sum_{K \in \mathcal{S}_{D}}(f, 1)_{K}=(f, 1)_{D}\right)$. If $D \in \mathcal{D}_{h}^{\text {ext }}$, this argument is replaced by the fact that we are free to choose the fluxes over the boundary sides.

For every $D \in \mathcal{D}_{h}$, we now have two equilibrated fluxes $\mathbf{t}_{1, D}$ and $\mathbf{t}_{2, D}$. Any $\mathbf{t}_{D}:=\alpha \mathbf{t}_{1, D}+$ $(1-\alpha) \mathbf{t}_{2, D}$, with a real parameter $\alpha$, obviously obeys Assumption 4.4 with $\mathcal{D}_{h}^{*}=\mathcal{D}_{h}$ and we can minimize $\eta_{D}:=\eta_{\mathrm{R}, D}+\eta_{\mathrm{DF}, D}$ as a function of the parameter $\alpha$. It turns out that it is much easier to minimize $\eta_{\mathrm{R}, D}^{2}+\eta_{\mathrm{DF}, D}^{2}$, as this is a quadratic function of $\alpha$, and the optimal value is easily found to be given by the equation

$$
\begin{aligned}
& \alpha\left(\left\|a^{-\frac{1}{2}}\left(\mathbf{t}_{1, D}-\mathbf{t}_{2, D}\right)\right\|_{D}^{2}+m_{D, a}^{2}\left\|\nabla \cdot\left(\mathbf{t}_{1, D}-\mathbf{t}_{2, D}\right)\right\|_{D}^{2}\right) \\
= & -\left(a^{\frac{1}{2}} \nabla p_{h}+a^{-\frac{1}{2}} \mathbf{t}_{2, D}, a^{-\frac{1}{2}}\left(\mathbf{t}_{1, D}-\mathbf{t}_{2, D}\right)\right)_{D} \\
& +m_{D, a}^{2}\left(f-\nabla \cdot \mathbf{t}_{2, D}, \nabla \cdot\left(\mathbf{t}_{1, D}-\mathbf{t}_{2, D}\right)\right)_{D} .
\end{aligned}
$$

Remark, however, that this value does not necessarily minimize $\eta_{D}$ but $\eta_{\mathrm{R}, D}^{2}+\eta_{\mathrm{DF}, D}^{2}$. For this reason, we finally propose as an estimator improving on those from the two previous sections

$$
\eta_{D}:=\min \left\{\eta_{D}\left(\mathbf{t}_{1, D}\right), \eta_{D}\left(\mathbf{t}_{2, D}\right), \eta_{D}\left(\alpha \mathbf{t}_{1, D}+(1-\alpha) \mathbf{t}_{2, D}\right)\right\} .
$$

Such an estimator will be locally efficient (and robust) whenever it is the case for $\eta_{D}\left(\mathbf{t}_{1, D}\right)$, which we recall only relies on the construction (4.8). The numerical experiments for this estimator are presented in Section 7.1.2.

The above recipe necessitates the construction of the two equilibrated fluxes $\mathbf{t}_{1, D}$ and $\mathbf{t}_{2, D}$ and the calculation of the parameter $\alpha$. In practice, it is often enough to construct $\mathbf{t}_{2, D}$ only and to take $\left.\mathbf{t}_{h}\right|_{D}=\mathbf{t}_{2, D}$, i.e., $\eta_{D}=\eta_{D}\left(\mathbf{t}_{2, D}\right)$ instead of evaluating the minimum in (4.9). This simplifies considerably the implementation and leads to computationally indistinguishable results.

\subsubsection{Construction of $t_{h}$ by mixed finite element approximations of local Neumann/ Dirichlet problems}

We adapt here to the present setting the approach of [28]. In context of a posteriori error estimation, solution of local Neumann problems can be traced back at least to [10].

For a given dual volume $D \in \mathcal{D}_{h}$, let

$$
\operatorname{RTN}_{N}\left(\mathcal{S}_{D}\right):=\left\{\mathbf{v}_{h} \in \mathbf{R T N}\left(\mathcal{S}_{D}\right) ; \mathbf{v}_{h} \cdot \mathbf{n}_{\sigma}=-\left\{\left\{a \nabla p_{h} \cdot \mathbf{n}_{\sigma}\right\}\right\}_{\omega} \quad \forall \sigma \in \mathcal{G}_{h}^{\text {int }} \cap \partial D\right\} .
$$

This is a space of Raviart-Thomas-Nédélec vector functions over the simplicial submesh $\mathcal{S}_{D}$ of the dual volume $D$ which are such that their normal components over that part of the boundary of $D$ which is inside $\Omega$ is given by the piecewise constant function $-\left\{\left\{a \nabla p_{h} \cdot \mathbf{n}_{\sigma}\right\}\right\}_{\omega}$. Note that it follows for any function in this space, by Assumption 4.9 and by the Green theorem, that Assumption (4.4) holds with $\mathcal{D}_{h}^{\text {int,* }}=\mathcal{D}_{h}^{\text {int }}$. As we will see below, one of the properties of the approach of this section is that we will eventually come to such $\mathbf{t}_{h}$ that Assumption (4.4) will hold more specifically with $\mathcal{D}_{h}^{\text {int,* }}=\mathcal{S}_{h}$ and $\mathcal{D}_{h}^{\text {ext,* }}=\emptyset$. 
Let $f_{h}$ be given by $(f, 1)_{K} /|K|$ for all $K \in \mathcal{S}_{h}$. We then define $\mathbf{t}_{h} \in \mathbf{R T N}\left(\mathcal{S}_{h}\right)$ by solving on each $D \in \mathcal{D}_{h}$ the following minimization problem:

$$
\left.\mathbf{t}_{h}\right|_{D}:=\arg \inf _{\mathbf{v}_{h} \in \mathbf{R T N}_{\mathrm{N}}\left(\mathcal{S}_{D}\right), \nabla \cdot \mathbf{v}_{h}=f_{h}}\left\|a^{\frac{1}{2}} \nabla p_{h}+a^{-\frac{1}{2}} \mathbf{v}_{h}\right\|_{D}
$$

Note that (a) we impose by a constraint that the residual estimators (4.3) will be very small, as $f-\nabla \cdot \mathbf{t}_{h}=f-f_{h}$; they will eventually disappear when $f=f_{h}$; (b) the equilibrated flux $\mathbf{t}_{h}$ that we find by (4.10) minimizes the diffusive flux estimator (4.2).

Define $\mathbf{R T N}_{\mathrm{N}, 0}\left(\mathcal{S}_{D}\right)$ as $\mathbf{R T N}_{\mathrm{N}}\left(\mathcal{S}_{D}\right)$ but with the normal flux condition $\mathbf{v}_{h} \cdot \mathbf{n}_{\sigma}=0$ for all the functions $\mathbf{v}_{h}$ from this space. Let $\mathbb{P}_{0}^{*}\left(\mathcal{S}_{D}\right)$ be spanned by piecewise constants on $\mathcal{S}_{D}$ with zero mean on $D$ when $D \in \mathcal{D}_{h}^{\text {int }}$; when $D \in \mathcal{D}_{h}^{\text {ext }}$, the mean value condition is not imposed. Then it is easy to show that (4.10) is equivalent to finding $\mathbf{t}_{h} \in \mathbf{R T N}_{\mathrm{N}}\left(\mathcal{S}_{D}\right)$ and $q_{h} \in \mathbb{P}_{0}^{*}\left(\mathcal{S}_{D}\right)$ such that

$$
\begin{aligned}
& \left(a^{-1} \mathbf{t}_{h}+\nabla p_{h}, \mathbf{v}_{h}\right)_{D}-\left(q_{h}, \nabla \cdot \mathbf{v}_{h}\right)_{D}=0 \quad \forall \mathbf{v}_{h} \in \mathbf{R T N}_{\mathrm{N}, 0}\left(\mathcal{S}_{D}\right), \\
& \left(\nabla \cdot \mathbf{t}_{h}, \phi_{h}\right)_{D}=\left(f, \phi_{h}\right)_{D} \quad \forall \phi_{h} \in \mathbb{P}_{0}^{*}\left(\mathcal{S}_{D}\right) \text {. }
\end{aligned}
$$

Note that (4.11a)-(4.11b) is the lowest-order Raviart-Thomas-Nédélec mixed finite element approximation of a local Neumann problem on the interior dual volumes $D \in \mathcal{D}_{h}^{\text {int }}$; the Neumann boundary condition is given by $-\left\{\left\{a \nabla p_{h} \cdot \mathbf{n}_{\sigma}\right\}\right\} \omega$. On the boundary dual volumes $D \in \mathcal{D}_{h}^{\text {ext }},(4.11 \mathrm{a})-$ (4.11b) is the lowest-order Raviart-Thomas-Nédélec mixed finite element approximation of a local problem where the same Neumann boundary condition is imposed on that part of the boundary of $D$ which lies inside $\Omega$ and the homogeneous Dirichlet boundary condition is imposed on the remaining part of the boundary of $D$. Note in particular that the function $-\left\{\left\{a \nabla p_{h} \cdot \mathbf{n}_{\sigma}\right\} \omega\right.$ on the boundary of each $D \in \mathcal{D}_{h}^{\text {int }}$ by Assumption 4.9 satisfies the Neumann compatibility condition, whence the existence and uniqueness of a solution of (4.11a)-(4.11b) follow. Theorem 4.5 and Corollary 4.6 can be used here with $\mathcal{D}_{h}^{\text {int,* }}=\mathcal{S}_{h}$ and $\mathcal{D}_{h}^{\text {ext,* }}=\emptyset$. A solution of the local linear system (4.11a)-(4.11b) on each $D \in \mathcal{D}_{h}$ is necessary in this approach but the numerical results of Sections 7.2.2-7.2.3 below reveal excellent.

The above presentation is done in the energy norm (2.10) setting. For the dual norm (2.11), we merely need to replace $\left(a^{-1} \mathbf{t}_{h}+\nabla p_{h}, \mathbf{v}_{h}\right)_{D}$ by $\left(\mathbf{t}_{h}+a \nabla p_{h}, \mathbf{v}_{h}\right)_{D}$ in (4.11a).

\subsection{Remarks and generalizations}

Remark 4.10 (Comparison with standard residual estimators). The estimates of Theorem 4.5 or Corollary 4.6 have three basic advantages in comparison with standard residual estimators, cf. Verfürth [54]. First of all, they feature no undetermined constant and deliver a guaranteed upper bound. Next, the classical residual estimator $h_{K}\left\|f+\nabla \cdot\left(a \nabla p_{h}\right)\right\|_{K}=h_{K}\|f\|_{K}$ is replaced by its improved version (4.3). Lastly, as it will be seen in Section 5 below, our estimates represent local lower bounds for the classical residual estimators. The improved behavior of our estimators over the classical one for the finite element method is numerically studied in [21].

Remark 4.11 (Comparison with the equilibrated residual method). In the equilibrated residual method, cf. [3], one searches equilibrated side fluxes expressing local conservativity over each $K \in \mathcal{T}_{h}$, by means of solution of local linear systems. Contrarily to this approach, our estimators are based on the immediately available conservativity of the finite element (and other) method(s) over the dual grids $\mathcal{D}_{h}$ (see Remark 3.13). On the other hand, we suggest herein the present approach only for lowest-order finite elements, whereas the approach of [3] works for any order. For the extension of the present approach to any order schemes (in the Stokes setting), we refer to [32]. Remark that a guaranteed and locally computable upper bound can also be obtained in the equilibrated residual method if the data oscillation term is separated as in [2]. 
Remark 4.12 (Comparison with the Zienkiewicz-Zhu averaging). Similarly as in the ZienkiewiczZhu [62] estimator, we look here for a smoothened (averaged) flux $\mathbf{t}_{h}$ and introduce the diffusive flux estimator $\eta_{\mathrm{R}, D}$ of the form (4.2) or (4.5). We, however, only impose $\mathbf{t}_{h} \in \mathbf{H}$ (div, $\Omega$ ), i.e., only the normal component continuity and not the continuity of the whole vector field $\mathbf{t}_{h}$. Also, our residual estimators $\eta_{\mathrm{R}, D}$, not present in the Zienkiewicz-Zhu setting, can become crucial on rough meshes or in the presence of the (discontinuous) material coefficient $a$ (recall that contrarily to the Zienkiewicz-Zhu setting, our estimates are guaranteed). We refer to other remarks to [31].

Remark 4.13 (Comparison with functional a posteriori estimates). Repin [46] or Korotov [35] use instead of Theorem 4.5 the estimate

$$
\left\|p-p_{h}\right\| \leq \frac{C_{\mathrm{F}, \Omega}^{1 / 2} h_{\Omega}}{c_{a, \Omega}^{1 / 2}}\left\|f-\nabla \cdot \mathbf{t}_{h}\right\|+\left\|a^{\frac{1}{2}} \nabla p_{h}+a^{-\frac{1}{2}} \mathbf{t}_{h}\right\|,
$$

which follows readily from Theorem 4.1 using the Cauchy-Schwarz inequality, the Friedrichs inequality, and the definition of the energy norm. Here $p$ is the weak solution given by (2.9), $p_{h} \in H_{0}^{1}(\Omega)$ and $\mathbf{t}_{h} \in \mathbf{H}(\operatorname{div}, \Omega)$ are arbitrary, $C_{\mathrm{F}, \Omega}$ is the constant from the Friedrichs inequality (2.7) with $D=\Omega$, and $h_{\Omega}$ is the diameter of $\Omega$. The advantage of such an approach is that no particular construction of $\mathbf{t}_{h} \in \mathbf{H}(\operatorname{div}, \Omega)$ has to be done and the estimate is fully schemeindependent. However, as no information from the computation is used, the first term on the right-hand side of (4.12) is in general too large by the presence of $h_{\Omega}$ instead of $h_{D}$ which we find

in Theorem 4.5. Additionally, the term $1 / c_{a, \Omega}^{1 / 2}$ is also unfavorable in comparison with $1 / c_{a, D}^{1 / 2}$ found in our estimates. Thus, a rather expensive global minimization is usually employed in the type of estimates of [46] or [35]. We present in Sections 7.1.1 and 7.2.1 below a comparison of the estimates of Theorem 4.5 and of the estimate of the form (4.12), when used without any minimization.

Remark 4.14 (Comparison with the estimator of Luce and Wohlmuth [39]). Our estimators are close to those of Luce and Wohlmuth [39], in particular in that we construct the dual mesh $\mathcal{D}_{h}$ and the second simplicial triangulation $\mathcal{S}_{h}$ and an equilibrated flux $\mathbf{t}_{h} \in \mathbf{R T N}\left(\mathcal{S}_{h}\right)$. One particular difference is that the construction of $\mathbf{t}_{h}$ by (4.8) with harmonic averaging, as shown in Section 5.1.1 below, leads to full robustness of our estimates with respect to discontinuous coefficients in the energy norm.

Remark 4.15 (Residual estimators and data oscillation). Note that whenever $f \in H^{1}(K)$ for all $K \in \mathcal{S}_{h}$, the residual estimators $\eta_{\mathrm{R}, D}$ in Section 4.3.4 (or those of Section 4.3.3 with $\mathbf{t}_{2, D}$ only) represent a contribution of higher order, as $\left\|f-f_{h}\right\|_{K} \leq 1 / \pi h_{K}\|\nabla f\|_{K}$ by the Poincaré inequality (2.6) (using the convexity of simplices). Moreover, if $f$ is piecewise constant on $\mathcal{S}_{h}$, they disappear completely.

\section{Efficiency and robustness of the a posteriori error estimates}

We prove here the (local) efficiency and, under appropriate conditions, robustness of our estimates. We first focus on the construction of the equilibrated flux by the direct prescription of Section 4.3.1 and present a robustness energy norm (2.10) result in case of discontinuities aligned with the dual meshes and use of harmonic averaging. Then robustness in the dual norm (2.11) without any special requirement is proven. Subsequently, similar results are given for the construction of $\mathbf{t}_{h}$ by the local Neumann/Dirichlet problems of Section 4.3.4. Finally, some generalizations are discussed. We once again proceed as generally as possible, without the definition of a particular numerical scheme. Note that, however, in contrast to Section 4, we need more properties of the equilibrated flux $\mathbf{t}_{h}$ than merely Assumption 4.4 or Assumption 4.9 to proceed. We will either assume (4.8) or (4.11a)-(4.11b). 


\subsection{Construction of $t_{h}$ by the direct prescription of Section 4.3.1}

We focus here on the equilibrated flux $\mathbf{t}_{h}$ defined in Section 4.3.1 by (4.8). More generally, $\mathbf{t}_{h}$ can also be given by the approaches of Section 4.3.2 or 4.3.3, as these estimators are by construction smaller or equal as that of Section 4.3.1. We first focus on the energy norm (2.10) setting and then pass to the dual norm (2.11) setting.

\subsubsection{Local efficiency and robustness of the energy norm estimate for harmonic weighting and dual mesh-aligned discontinuities}

The result of this section is given in the energy norm (2.10) and only applies to the case where $a$ is piecewise constant on $\mathcal{D}_{h}$ and $\omega$ in Assumption 4.9 represents harmonic weights (2.5).

Theorem 5.1 (Local efficiency and robustness of the energy norm estimate for harmonic weighting and dual mesh-aligned discontinuities). Let a be piecewise constant on $\mathcal{D}_{h}$, let $f$ be a piecewise polynomial of degree $m$ on $\mathcal{S}_{h}$, let $p$ be the weak solution of problem (1.1a)-(1.1b), and let $p_{h} \in X_{h}^{0}$ satisfy Assumption 4.9 with the harmonic averaging weights (2.5). Let $\mathcal{S}_{h}$ be shape-regular with the constant $\kappa_{\mathcal{S}}$. Let finally $\mathbf{t}_{h}$ be given by (4.8), $\eta_{\mathrm{DF}, D}$ by $(4.2), \eta_{\mathrm{R}, D}$ by $(4.3)$, and $\mathcal{D}_{h}^{*}=\mathcal{D}_{h}$ in Theorem 4.5. Then, for each $D \in \mathcal{D}_{h}$, there holds

$$
\begin{aligned}
\eta_{\mathrm{DF}, D} & \leq C\|\mid\| p-p_{h} \|_{\mathcal{T}_{V_{D}}}, \\
\eta_{\mathrm{R}, D} & \leq \tilde{C}\left\|p-p_{h}\right\|_{\mathcal{T}_{V_{D}}},
\end{aligned}
$$

where the constant $C$ depends only on $d, \kappa_{\mathcal{S}}$, and $m$ and $\tilde{C}$ in addition depends on $C_{\mathrm{P}, D}$ if $D \in \mathcal{D}_{h}^{\text {int }}$ or $C_{\mathrm{F}, D, \partial \Omega}$ if $D \in \mathcal{D}_{h}^{\text {ext }}$.

Recall that $\mathcal{T}_{V_{D}}$ in (5.1a) and (5.1b) stand for all the elements of the original simplicial mesh $\mathcal{T}_{h}$ sharing the vertex $V_{D}$ associated with the dual volume $D \in \mathcal{D}_{h}$. The proof of Theorem 5.1 is decomposed into two parts. For $\eta_{\mathrm{DF}, D}$, Lemma 5.2 shows that the construction (4.8) implies that the normal components of $\mathbf{t}_{h}$ differ from those of $a \nabla p_{h}$ by the jumps of $a \nabla p_{h} \cdot \mathbf{n}_{\sigma}$. The latter are a part of residual estimators and are therefore known to be bounded by the error. The second estimator, $\eta_{\mathrm{R}, D}$, is then efficient due to a complementarity argument as shown in Lemma 5.3.

Lemma 5.2 (Local efficiency of the diffusive flux estimator). Let the assumptions of Theorem 5.1 be verified. Then (5.1a) holds true.

Proof. The proof follows the techniques of [54] and [27]. Recall first the standard estimate

$$
\left\|\mathbf{v}_{h}\right\|_{K}^{2} \leq C h_{K} \sum_{\sigma \in \mathcal{E}_{K}}\left\|\mathbf{v}_{h} \cdot \mathbf{n}_{\sigma}\right\|_{\sigma}^{2}
$$

valid for each $\mathbf{v}_{h} \in \mathbf{R T N}(K)$ and any simplex $K$. Here, and similarly in the rest of the proof, the constant $C$, not necessarily the same at each occurrence, depends only on $d, \kappa_{\mathcal{S}}$, and $m$.

Let now $K$ be an arbitrary element in the simplicial mesh $\mathcal{S}_{D}$ of a given $D \in \mathcal{D}_{h}$ and let us set $\mathbf{v}_{h}=a \nabla p_{h}+\mathbf{t}_{h}$. Recall that we suppose that $\mathbf{t}_{h}$ is given by (4.8), with the harmonic averaging weights (2.5). Let $\sigma \in \mathcal{E}_{K} \cap \mathcal{G}_{h}^{\text {ext }}$. Then $\left.\left(a \nabla p_{h}+\mathbf{t}_{h}\right)\right|_{K} \cdot \mathbf{n}_{\sigma}=0$ by (2.2c). Let $\sigma \in \mathcal{E}_{K} \cap \mathcal{G}_{h}^{\text {int }}$. Then

$$
\left.\left(a \nabla p_{h}+\mathbf{t}_{h}\right)\right|_{K} \cdot \mathbf{n}_{\sigma}=\left.\left(a \nabla p_{h} \cdot \mathbf{n}_{\sigma}\right)\right|_{K}-\left\{\left\{a \nabla p_{h} \cdot \mathbf{n}_{\sigma}\right\}\right\}_{\omega}=\mathbf{n}_{\sigma} \cdot \mathbf{n}_{K} \omega_{L, \sigma} \llbracket a \nabla p_{h} \cdot \mathbf{n}_{\sigma} \rrbracket,
$$

where $L$ denotes the neighboring element to $K$ across the side $\sigma$. Here $\mathbf{n}_{\sigma} \cdot \mathbf{n}_{K}= \pm 1$ is only used as a sign determination. Using these developments, we come to

$$
\left\|a^{\frac{1}{2}} \nabla p_{h}+a^{-\frac{1}{2}} \mathbf{t}_{h}\right\|_{K}^{2}=a_{K}^{-1}\left\|\mathbf{v}_{h}\right\|_{K}^{2} \leq C a_{K}^{-1} h_{K} \sum_{\sigma \in \mathcal{E}_{K} \cap \mathcal{G}_{h}^{\text {int }}}\left\|\omega_{L, \sigma} \llbracket a \nabla p_{h} \cdot \mathbf{n}_{\sigma} \rrbracket\right\|_{\sigma}^{2} .
$$


Let us now consider a fixed $\sigma=\sigma_{K, L} \in \mathcal{E}_{K} \cap \mathcal{G}_{h}^{\text {int }}$. The estimate

$$
h_{K}^{\frac{1}{2}}\left\|\llbracket a \nabla p_{h} \cdot \mathbf{n}_{\sigma} \rrbracket\right\|_{\sigma} \leq C \sum_{M \in\{K, L\}} a_{M}^{\frac{1}{2}}\left\|p-p_{h}\right\| \|_{M}
$$

is standard, see [54]. Recall from [54] that the proof uses the side and element bubble functions, which enable to discard the boundary terms in the application of the Green theorem, the inverse inequality, and the equivalence of norms on finite-dimensional spaces (the assumption that $f$ is a piecewise polynomial and the fact that the side and element bubble functions are piecewise polynomials are crucial). It then follows that

$$
\omega_{L, \sigma} a_{K}^{-\frac{1}{2}} h_{K}^{\frac{1}{2}}\left\|\llbracket a \nabla p_{h} \cdot \mathbf{n}_{\sigma} \rrbracket\right\|_{\sigma} \leq C \sum_{M \in\{K, L\}} \omega_{L, \sigma} a_{K}^{-\frac{1}{2}} a_{M}^{\frac{1}{2}}\left\|p-p_{h}\right\| \|_{M} .
$$

Thanks to the definition (2.2) of $\omega_{L, \sigma}, \omega_{L, \sigma} a_{K}^{-\frac{1}{2}} a_{M}^{\frac{1}{2}}=\omega_{L, \sigma} \leq 1$ if $M=K$ and by (2.5), $\omega_{L, \sigma} a_{K}^{-\frac{1}{2}} a_{M}^{\frac{1}{2}}=$ $a_{K}\left(a_{K}+a_{L}\right)^{-1} a_{K}^{-\frac{1}{2}} a_{L}^{\frac{1}{2}} \leq \frac{1}{2}$ if $M=L$, using the inequality $2 a b \leq a^{2}+b^{2}$.

Thus, using the above results,

$$
\begin{aligned}
\eta_{\mathrm{DF}, D}^{2} & =\sum_{K \in \mathcal{S}_{D}}\left\|a^{\frac{1}{2}} \nabla p_{h}+a^{-\frac{1}{2}} \mathbf{t}_{h}\right\|_{K}^{2} \\
& \leq C \sum_{K \in \mathcal{S}_{D}} \sum_{\sigma_{K, L} \in \mathcal{E}_{K} \cap \mathcal{G}_{h}^{\text {int }}} a_{K}^{-1} h_{K} \omega_{L, \sigma_{K, L}}^{2}\left\|\llbracket a \nabla p_{h} \cdot \mathbf{n}_{\sigma_{K, L}} \rrbracket\right\|_{\sigma_{K, L}}^{2} \\
& \leq C \sum_{K \in \mathcal{S}_{D}} \sum_{\sigma_{K, L} \in \mathcal{E}_{K} \cap \mathcal{G}_{h}^{\text {int }}} \sum_{M \in\{K, L\}}\left\|p-p_{h}\right\|\left\|_{M}^{2} \leq C\right\| p-p_{h} \|_{\mathcal{T}_{V_{D}}}^{2},
\end{aligned}
$$

which was to be proved.

Lemma 5.3 (Local efficiency of the residual estimator). Let the assumptions of Theorem 5.1 be verified. Then (5.1b) holds true.

Proof. Let us consider a fixed dual volume $D \in \mathcal{D}_{h}$. First,

$$
\left\|f-\nabla \cdot \mathbf{t}_{h}\right\|_{K} \leq C a_{K}^{\frac{1}{2}} h_{K}^{-1}\left\|a^{\frac{1}{2}} \nabla p+a^{-\frac{1}{2}} \mathbf{t}_{h}\right\|_{K}
$$

for each $K \in \mathcal{S}_{D}$, with $C$ depending only on $d, \kappa_{\mathcal{S}}$, and $m$, follows standardly by using the element bubble function, the equivalence of norms on finite-dimensional spaces, definition (2.9) of the weak solution, the Green theorem, the Cauchy-Schwarz inequality, definition (2.10) of the energy norm, and the inverse inequality, cf. [54] or [57, Lemma 7.6]. Hence

$$
\left\|f-\nabla \cdot \mathbf{t}_{h}\right\|_{D} \leq C C_{a, D}^{\frac{1}{2}} h_{D}^{-1}\left\|a^{\frac{1}{2}} \nabla p+a^{-\frac{1}{2}} \mathbf{t}_{h}\right\|_{D}
$$

holds true, using the fact that $h_{D} / \min _{K \in \mathcal{S}_{D}} h_{K}$ is bounded by the shape-regularity of $\mathcal{S}_{h}$. Thus

$$
h_{D} c_{a, D}^{-\frac{1}{2}}\left\|f-\nabla \cdot \mathbf{t}_{h}\right\|_{D} \leq C c_{a, D}^{-\frac{1}{2}} C_{a, D}^{\frac{1}{2}}\left\|a^{\frac{1}{2}} \nabla p+a^{-\frac{1}{2}} \mathbf{t}_{h}\right\|_{D} .
$$

Next note that $c_{a, D}^{-\frac{1}{2}} C_{a, D}^{\frac{1}{2}}=1$ for $a$ piecewise constant on $\mathcal{D}_{h}$. Finally,

$$
\left\|a^{\frac{1}{2}} \nabla p+a^{-\frac{1}{2}} \mathbf{t}_{h}\right\|_{D} \leq\left\|p-p_{h}\right\|\left\|_{D}+\right\| a^{\frac{1}{2}} \nabla p_{h}+a^{-\frac{1}{2}} \mathbf{t}_{h} \|_{D}
$$

using the triangle inequality, which concludes the proof by virtue of the previously proved estimate (5.1a). 


\subsubsection{Global efficiency and robustness of the dual norm estimate}

The result of this section is given in the dual norm (2.11) and applies without any restriction on the distribution of the discontinuities (they can be piecewise constant on $\mathcal{T}_{h}$ or on $\mathcal{D}_{h}$ ) or type of averaging (both arithmetic and harmonic averaging is allowed) in Assumption 4.9.

Theorem 5.4 (Global efficiency and robustness of the dual norm a posteriori error estimates). Let $f$ be a piecewise polynomial of degree $m$ on $\mathcal{S}_{h}$, let $p$ be the weak solution of problem (1.1a)-(1.1b), and let $p_{h} \in X_{h}^{0}$ satisfy Assumption 4.9 with any weights satisfying (2.2). Let $\mathcal{S}_{h}$ be shape-regular with the constant $\kappa_{\mathcal{S}}$. Let finally $\mathbf{t}_{h}$ be given by (4.8), $\eta_{\mathrm{DF}, D}$ by (4.5), $\eta_{\mathrm{R}, D}$ by (4.6), and $\mathcal{D}_{h}^{*}=\mathcal{D}_{h}$ in Corollary 4.6. Then, there holds

$$
\left\{\sum_{D \in \mathcal{D}_{h}}\left(\eta_{\mathrm{DF}, D}+\eta_{\mathrm{R}, D}\right)^{2}\right\}^{\frac{1}{2}} \leq C \mid\left\|p-p_{h}\right\|_{\#},
$$

where the constant $C$ depends only on $d, \kappa_{\mathcal{S}}, m$, and $C_{\mathrm{P}, D}$ for $D \in \mathcal{D}_{h}^{\text {int }}$ and $C_{\mathrm{F}, D, \partial \Omega}$ for $D \in \mathcal{D}_{h}^{\text {ext }}$.

Proof. Throughout this proof, $C$ denotes a generic constant with the dependencies indicated in the announcement of the theorem, possibly different at different occurrences. Let $K \in \mathcal{S}_{D}, D \in \mathcal{D}_{h}$ be given. Adding and subtracting $\nabla \cdot\left(a \nabla p_{h}\right)$, using the triangle inequality, the fact that $h_{D} \leq C h_{K}$ by the shape-regularity of $\mathcal{D}_{h}$, and the inverse inequality, we have

$$
\begin{aligned}
C_{\mathrm{P}, D}^{\frac{1}{2}} h_{D}\left\|f-\nabla \cdot \mathbf{t}_{h}\right\|_{K} & \leq C_{\mathrm{P}, D}^{\frac{1}{2}} h_{D}\left(\left\|f+\nabla \cdot\left(a \nabla p_{h}\right)\right\|_{K}+\left\|\nabla \cdot\left(a \nabla p_{h}+\mathbf{t}_{h}\right)\right\|_{K}\right) \\
& \leq C h_{K}\left\|f+\nabla \cdot\left(a \nabla p_{h}\right)\right\|_{K}+C\left\|a \nabla p_{h}+\mathbf{t}_{h}\right\|_{K} .
\end{aligned}
$$

Using (5.2), (4.8), (5.3), and (2.2), we obtain

$$
\left\|a \nabla p_{h}+\mathbf{t}_{h}\right\|_{K}^{2} \leq C h_{K} \sum_{\sigma \in \mathcal{E}_{K} \cap \mathcal{G}_{h}^{\text {int }}}\left\|\llbracket a \nabla p_{h} \cdot \mathbf{n}_{\sigma} \rrbracket\right\|_{\sigma}^{2}
$$

(note that in both cases that $a$ is piecewise constant on $\mathcal{T}_{h}$ or that $a$ is piecewise constant on $\mathcal{D}_{h}$, $a$ is piecewise constant on $\mathcal{S}_{h}$ ). Combining the two above estimates,

$$
\sum_{D \in \mathcal{D}_{h}}\left(\eta_{\mathrm{DF}, D}+\eta_{\mathrm{R}, D}\right)^{2} \leq C\left(\sum_{K \in \mathcal{S}_{h}} h_{K}^{2}\left\|f+\nabla \cdot\left(a \nabla p_{h}\right)\right\|_{K}^{2}+\sum_{\sigma \in \mathcal{G}_{h}^{\text {int }}} h_{\sigma}\left\|\llbracket a \nabla p_{h} \cdot \mathbf{n}_{\sigma} \rrbracket\right\|_{\sigma}^{2}\right) .
$$

Note that this means that the present estimates represent a lower bound for the standard residual ones (cf. [54]). The rest of the proof is based on the tools from [55].

We next prove that

$$
\left\{\sum_{K \in \mathcal{S}_{h}} h_{K}^{2}\left\|f+\nabla \cdot\left(a \nabla p_{h}\right)\right\|_{K}^{2}\right\}^{\frac{1}{2}} \leq C \mid\left\|p-p_{h}\right\|_{\#} \cdot
$$

Let $K \in \mathcal{S}_{h}$. Denote by $\psi_{K}$ the element bubble function (cf. [54]). Recall that this function is given as the product of the $d+1$ affine functions which take the value 1 in one vertex and value 0 in the other vertices of the element $K$, scaled in such a way that $\left\|\psi_{K}\right\|_{\infty, K}=1$. Set $v_{K}:=\left.\left(f+\nabla \cdot\left(a \nabla p_{h}\right)\right)\right|_{K}$. By the equivalence of norms on finite-dimensional spaces, the above definition of the bubble function, and definition (2.9) of the weak solution, we have, see [54],

$$
\left\|v_{K}\right\|_{K}^{2} \leq C\left(a \nabla\left(p-p_{h}\right), \nabla\left(\psi_{K} v_{K}\right)\right)_{K} .
$$


Next, by the inverse inequality and the fact that $\left\|\psi_{K}\right\|_{\infty, K}=1$,

$$
h_{K}^{2}\left\|\nabla\left(\psi_{K} v_{K}\right)\right\|_{K} \leq C h_{K}\left\|v_{K}\right\|_{K} .
$$

Set $\left.\lambda\right|_{K}=h_{K}^{2} \psi_{K} v_{K}$ and note that $\lambda \in H_{0}^{1}(\Omega)$. Using the two above inequalities,

$$
\begin{aligned}
\sum_{K \in \mathcal{S}_{h}} h_{K}^{2}\left\|v_{K}\right\|_{K}^{2} & \leq C \sum_{K \in \mathcal{S}_{h}} h_{K}^{2}\left(a \nabla\left(p-p_{h}\right), \nabla\left(\psi_{K} v_{K}\right)\right)_{K}=C \frac{\mathcal{B}\left(p-p_{h}, \lambda\right)}{\|\nabla \lambda\|}\|\nabla \lambda\| \\
& \leq C\left\|p-p_{h}\right\|\left\|_{\#}\left\{\sum_{K \in \mathcal{S}_{h}} h_{K}^{4}\left\|\nabla\left(\psi_{K} v_{K}\right)\right\|_{K}^{2}\right\}^{\frac{1}{2}} \leq C\right\| p-p_{h}\|\|_{\#}\left\{\sum_{K \in \mathcal{S}_{h}} h_{K}^{2}\left\|v_{K}\right\|_{K}^{2}\right\}^{\frac{1}{2}}
\end{aligned}
$$

employing also the definition (2.11) of the dual norm and the Cauchy-Schwarz inequality. Thus (5.5) is proved.

The final point of the proof is to show that

$$
\left\{\sum_{\sigma \in \mathcal{G}_{h}^{\text {int }}} h_{\sigma}\left\|\llbracket a \nabla p_{h} \cdot \mathbf{n}_{\sigma} \rrbracket\right\|_{\sigma}^{2}\right\}^{\frac{1}{2}} \leq C\|\| p-p_{h} \|_{\#} .
$$

For $\sigma \in \mathcal{G}_{h}^{\text {int }}$, set $\left.v\right|_{\sigma}:=\llbracket a \nabla p_{h} \cdot \mathbf{n}_{\sigma} \rrbracket$; we keep the same notation for the lifting of $\left.v\right|_{\sigma}$ to the two simplices $K$ and $L$ sharing the side $\sigma$. Let $\psi_{\sigma}$ be the side bubble function (cf. once again [54]). Then there holds

$$
\begin{aligned}
\left\|v_{\sigma}\right\|_{\sigma}^{2} & \leq C\left\langle v_{\sigma}, \psi_{\sigma} v_{\sigma}\right\rangle_{\sigma}, \\
\left\|\psi_{\sigma} v_{\sigma}\right\|_{K} & \leq C h_{\sigma}^{\frac{1}{2}}\left\|v_{\sigma}\right\|_{\sigma} .
\end{aligned}
$$

Set $\lambda:=\sum_{\sigma \in \mathcal{G}_{h}^{\text {int }}} h_{\sigma} \psi_{\sigma} v_{\sigma}$. Note that $\lambda \in H_{0}^{1}(\Omega)$, as only the interior sides appear in the sum. Finally, note that by the second of the above inequalities,

$$
\|\lambda\|_{K} \leq \sum_{\sigma \in \mathcal{E}_{K} \cap \mathcal{G}_{h}^{\text {int }}} h_{\sigma}\left\|\psi_{\sigma} v_{\sigma}\right\|_{K} \leq C \sum_{\sigma \in \mathcal{E}_{K} \cap \mathcal{G}_{h}^{\text {int }}} h_{\sigma}^{\frac{3}{2}}\left\|v_{\sigma}\right\|_{\sigma} .
$$

Using the above inequalities and the Green theorem,

$$
\begin{aligned}
& \sum_{\sigma \in \mathcal{G}_{h}^{\text {int }}} h_{\sigma}\left\|v_{\sigma}\right\|_{\sigma}^{2} \\
\leq & C \sum_{\sigma \in \mathcal{G}_{h}^{\text {int }}}\left\langle\llbracket a \nabla p_{h} \cdot \mathbf{n}_{\sigma} \rrbracket, \lambda\right\rangle_{\sigma}=C \sum_{K \in \mathcal{S}_{h}}\left\{\left(f+\nabla \cdot\left(a \nabla p_{h}\right), \lambda\right)_{K}-\left(a \nabla\left(p-p_{h}\right), \nabla \lambda\right)_{K}\right\} \\
\leq & C\left\|p-p_{h}\right\|\left\|_{\#}\right\| \nabla \lambda \|+C\left\{\sum_{K \in \mathcal{S}_{h}} h_{K}^{2}\left\|f+\nabla \cdot\left(a \nabla p_{h}\right)\right\|_{K}^{2}\right\}^{\frac{1}{2}}\left\{\sum_{K \in \mathcal{S}_{h}} h_{K}^{-2}\|\lambda\|_{K}^{2}\right\}^{\frac{1}{2}} \\
\leq & C\left\|p-p_{h}\right\|_{\#}\left\{\sum_{K \in \mathcal{S}_{h}} h_{K}^{-2}\|\lambda\|_{K}^{2}\right\}^{\frac{1}{2}} \leq C\left\|p-p_{h}\right\| \|_{\#}\left\{\sum_{\sigma \in \mathcal{G}_{h}^{\text {int }}} h_{\sigma}\left\|v_{\sigma}\right\|_{\sigma}^{2}\right\}^{\frac{1}{2}},
\end{aligned}
$$

where we have also employed (5.5), the inverse inequality, and the Cauchy-Schwarz inequality. Thus (5.6) is proved. 


\subsection{Construction of $\mathrm{t}_{h}$ by the local Neumann/Dirichlet problems of Section 4.3.4}

We focus here on the equilibrated flux $\mathbf{t}_{h}$ defined in Section 4.3.4 by (4.11a)-(4.11b). As in the previous section, we first treat the energy norm (2.10) setting and then pass to the dual norm (2.11) setting.

\subsubsection{Local efficiency of the energy norm estimate}

The following result is given in the energy norm (2.10) and applies without any restriction on the distribution of discontinuities or type of averaging in Assumption 4.9.

Theorem 5.5 (Efficiency of the energy estimates by the local Neumann/Dirichlet problems). Let $f$ be a piecewise polynomial of degree $m$ on $\mathcal{S}_{h}$, let $p$ be the weak solution of problem (1.1a)-(1.1b), and let $p_{h} \in X_{h}^{0}$ satisfy Assumption 4.9 with any weights satisfying (2.2). Let $\mathcal{S}_{h}$ be shape-regular with the constant $\kappa_{\mathcal{S}}$. Let finally $\mathbf{t}_{h}$ be given by (4.11a)-(4.11b), $\eta_{\mathrm{DF}, D}$ by (4.2), $\eta_{\mathrm{R}, D}$ by (4.3), and $\mathcal{D}_{h}^{\mathrm{int}, *}=\mathcal{S}_{h}$ and $\mathcal{D}_{h}^{\mathrm{ext}, *}=\emptyset$ in Theorem 4.5. Then, for each $D \in \mathcal{D}_{h}$, there holds

$$
\begin{aligned}
\eta_{\mathrm{R}, K}= & C_{\mathrm{P}, K}^{\frac{1}{2}} \frac{h_{K}}{c_{a, K}^{\frac{1}{2}}}\left\|f-f_{h}\right\|_{K} \quad \forall K \in \mathcal{S}_{D}, \\
\left\{\sum_{K \in \mathcal{S}_{D}} \eta_{\mathrm{DF}, K}^{2}\right\}^{\frac{1}{2}} \leq & C c_{a, D}^{-\frac{1}{2}}\left(\left\{\sum_{K \in \mathcal{S}_{D}} h_{K}^{2}\left\|f_{h}+\nabla \cdot\left(a \nabla p_{h}\right)\right\|_{K}^{2}\right\}^{\frac{1}{2}}\right. \\
& \left.+\left\{\sum_{K \in \mathcal{S}_{D}} \sum_{\sigma \in \mathcal{E}_{K} \cap \mathcal{G}_{h}^{\text {int }}} h_{\sigma}\left\|\llbracket a \nabla p_{h} \cdot \mathbf{n}_{\sigma} \rrbracket\right\|_{\sigma}^{2}\right\}^{\frac{1}{2}}\right) \\
\leq & C c_{a, D}^{-\frac{1}{2}} C_{a, \mathcal{T}_{V_{D}}}^{\frac{1}{2}}\left\|p-p_{h}\right\| \|_{\mathcal{T}_{V_{D}}},
\end{aligned}
$$

where the constant $C$ depends only on $d, \kappa_{\mathcal{S}}$, and $m$.

Proof. The result (5.7) is an immediate consequence of equation (4.11b). It thus remains to show (5.8).

Let $D \in \mathcal{D}_{h}$ be fixed. We need a hybridized version of (4.11a)-(4.11b), cf. [16, 49]. Therein, equation (4.11a) is replaced by

$$
\left(a^{-1} \mathbf{t}_{h}+\nabla p_{h}, \mathbf{v}_{h}\right)_{D}-\left(q_{h}, \nabla \cdot \mathbf{v}_{h}\right)_{D}+\sum_{K \in \mathcal{S}_{D}}\left\langle\mathbf{v}_{h} \cdot \mathbf{n}_{K}, \lambda_{h}\right\rangle_{\partial K}=0 \quad \forall \mathbf{v}_{h} \in \mathbf{R T N}_{\mathrm{N}, 0}^{*}\left(\mathcal{S}_{D}\right) ;
$$

$\mathbf{R T N}_{\mathrm{N}, 0}^{*}\left(\mathcal{S}_{D}\right)$ is the same space as $\mathbf{R T N}_{\mathrm{N}, 0}\left(\mathcal{S}_{D}\right)$ of Section 4.3 .4 with, however, no normal trace continuity constraint; $\lambda_{h}$ is the Lagrange multiplier, a piecewise constant function on the sides $\mathcal{G}_{D}$ of the simplicial patch $\mathcal{S}_{D}$ (we set $\lambda_{h}=0$ on $\partial \Omega$ ). We have introduced this hybridized version so as to be able to set $\mathbf{v}_{h}=\mathbf{t}_{h}+a \nabla p_{h}$ therein. This leads to

$$
\begin{aligned}
\left\|a^{\frac{1}{2}} \nabla p_{h}+a^{-\frac{1}{2}} \mathbf{t}_{h}\right\|_{D}^{2}= & \left(q_{h}, f_{h}+\nabla \cdot\left(a \nabla p_{h}\right)\right)_{D}-\sum_{\sigma \in \mathcal{G}_{D}^{\text {int }}}\left\langle\llbracket a \nabla p_{h} \cdot \mathbf{n}_{\sigma} \rrbracket, \lambda_{h}\right\rangle_{\sigma} \\
& -\sum_{\sigma \in \mathcal{G}_{D}^{\text {ext }}}\left\langle\left.\left(a \nabla p_{h} \cdot \mathbf{n}_{D}\right)\right|_{D}-\left\{\left\{a \nabla p_{h} \cdot \mathbf{n}_{D}\right\}\right\}_{\omega}, \lambda_{h}\right\rangle_{\sigma},
\end{aligned}
$$

using that $\nabla \cdot \mathbf{t}_{h}=f_{h}$ by $(4.11 \mathrm{~b})$, that the normal trace of $\mathbf{t}_{h}$ is continuous across $\sigma \in \mathcal{G}_{D}^{\text {int }}$, the interior sides of the patch $\mathcal{S}_{D}$, and the fact that $\mathbf{t}_{h} \in \mathbf{R T N}_{\mathrm{N}}\left(\mathcal{S}_{D}\right)$, which fixes its normal component on $\sigma \in \mathcal{G}_{D}^{\text {ext }}$, the boundary sides of the patch $\mathcal{S}_{D}$. 
We next employ the approach of [57, Section 4.1] (cf. also [6, 5]): there exists a postprocessing $\tilde{q}_{h} \in M\left(\mathcal{S}_{D}\right)$ of $q_{h}$ such that

$$
\begin{array}{ll}
-a \nabla \tilde{q}_{h}=\mathbf{t}_{h}+a \nabla p_{h} & \forall K \in \mathcal{S}_{D}, \\
\frac{\left(\tilde{q}_{h}, 1\right)_{K}}{|K|}=\left.q_{h}\right|_{K} & \forall K \in \mathcal{S}_{D}, \\
\frac{\left\langle\tilde{q}_{h}, 1\right\rangle_{\sigma}}{|\sigma|}=\left.\lambda_{h}\right|_{\sigma} & \forall \sigma \in \mathcal{G}_{D} .
\end{array}
$$

Here, $M\left(\mathcal{S}_{D}\right)$ is a space of particular piecewise polynomials on $\mathcal{S}_{D}$ of total degree $\leq 2$. Let hereafter $C$ be a generic constant only dependent on $d$ and $\kappa_{\mathcal{S}}$, possibly different at different occurrences. Let $D \in \mathcal{D}_{h}^{\text {int }}$. Then $\left(q_{h}, 1\right)_{D}=0$, as $q_{h} \in \mathbb{P}_{0}^{*}\left(\mathcal{S}_{D}\right)$, see (4.11a)-(4.11b). Thus, by $(5.10 \mathrm{~b}),\left(\tilde{q}_{h}, 1\right)_{D} /|D|=0$, i.e., the mean value of $\tilde{q}_{h}$ over $D$ is also zero. Let $D \in \mathcal{D}_{h}^{\text {ext }}$. Then $\lambda_{h}=0$ on $\partial D \cap \partial \Omega$. Consequently, by (5.10c), $\left\langle\tilde{q}_{h}, 1\right\rangle_{\sigma} /|\sigma|=0$, i.e., the mean values of $\tilde{q}_{h}$ over the sides lying in $\partial \Omega$ are zero when $D \in \mathcal{D}_{h}^{\text {ext }}$. Thus, for both $D \in \mathcal{D}_{h}^{\text {int }}$ and $D \in \mathcal{D}_{h}^{\text {ext }}$, we have the Poincaré/Friedrichs inequality $\left\|\tilde{q}_{h}\right\| \leq C h_{D}\left\|\nabla \tilde{q}_{h}\right\|_{D}$, cf. [56]. Employing also the inverse inequality $\left\|\tilde{q}_{h}\right\|_{\sigma} \leq C h_{\sigma}^{-\frac{1}{2}}\left\|\tilde{q}_{h}\right\|_{K}$ for any $K$ sharing a side $\sigma \in \mathcal{G}_{D}$, the Cauchy-Schwarz inequality, and the facts that $h_{D} / \min _{K \in \mathcal{S}_{D}} h_{K}$ and the number of elements $K$ in $\mathcal{S}_{D}$ are bounded by the shape-regularity of $\mathcal{S}_{h}$, we infer from (5.9), (5.3), and (2.2)

$$
\begin{aligned}
& \left\|a^{\frac{1}{2}} \nabla p_{h}+a^{-\frac{1}{2}} \mathbf{t}_{h}\right\|_{D}^{2} \\
= & \left(\tilde{q}_{h}, f_{h}+\nabla \cdot\left(a \nabla p_{h}\right)\right)_{D}-\sum_{\sigma \in \mathcal{G}_{D}^{\text {int }}}\left\langle\llbracket a \nabla p_{h} \cdot \mathbf{n}_{\sigma} \rrbracket, \tilde{q}_{h}\right\rangle_{\sigma} \\
& -\sum_{\sigma \in \mathcal{G}_{D}^{\text {ext }}}\left\langle\left.\left(a \nabla p_{h} \cdot \mathbf{n}_{D}\right)\right|_{D}-\left\{\left\{a \nabla p_{h} \cdot \mathbf{n}_{D}\right\}\right\} \omega, \tilde{q}_{h}\right\rangle_{\sigma}, \\
\leq & \left\|\tilde{q}_{h}\right\|_{D}\left\|f_{h}+\nabla \cdot\left(a \nabla p_{h}\right)\right\|_{D}+C\left\{\sum_{K \in \mathcal{S}_{D}} \sum_{\sigma \in \mathcal{E}_{K} \cap \mathcal{G}_{h}^{\text {int }}} h_{\sigma}^{-1}\left\|\llbracket a \nabla p_{h} \cdot \mathbf{n}_{\sigma} \rrbracket\right\|_{\sigma}^{2}\right\}^{\frac{1}{2}}\left\|\tilde{q}_{h}\right\|_{D} \\
\leq & C\left\|\nabla \tilde{q}_{h}\right\|_{D}\left(\left\{\sum_{K \in \mathcal{S}_{D}} h_{K}^{2}\left\|f_{h}+\nabla \cdot\left(a \nabla p_{h}\right)\right\|_{K}^{2}\right\}^{\frac{1}{2}}+\left\{\sum_{K \in \mathcal{S}_{D}} \sum_{\sigma \in \mathcal{E}_{K} \cap \mathcal{G}_{h}^{\text {int }}} h_{\sigma}\left\|\llbracket a \nabla p_{h} \cdot \mathbf{n}_{\sigma} \rrbracket\right\|_{\sigma}^{2}\right\}^{\frac{1}{2}}\right) .
\end{aligned}
$$

The assertion follows from (5.10a) while scaling by $c_{a, D}^{-\frac{1}{2}}$ and dividing by $\left\|a^{\frac{1}{2}} \nabla p_{h}+a^{-\frac{1}{2}} \mathbf{t}_{h}\right\|_{D}$ and using the results of the proofs of Theorems 5.1 and 5.4 .

\subsubsection{Global efficiency and robustness of the dual norm estimate}

For completeness, we also include the following result, given in the dual norm (2.11). This result applies without any restriction on the distribution of discontinuities or type of averaging in Assumption 4.9. The proof is an easy consequence of the previous results.

Corollary 5.6 (Efficiency of the dual estimates by the local Neumann/Dirichlet problems). Let $f$ be a piecewise polynomial of degree $m$ on $\mathcal{S}_{h}$, let $p$ be the weak solution of problem (1.1a)-(1.1b), and let $p_{h} \in X_{h}^{0}$ satisfy Assumption 4.9 with any weights satisfying $(2.2)$. Let $\mathcal{S}_{h}$ be shape-regular with the constant $\kappa_{\mathcal{S}}$. Let finally $\mathbf{t}_{h}$ be given by (4.11a)-(4.11b), $\eta_{\mathrm{DF}, D}$ by (4.5), $\eta_{\mathrm{R}, D}$ by (4.6), 
and $\mathcal{D}_{h}^{\text {int,* }}=\mathcal{S}_{h}$ and $\mathcal{D}_{h}^{\text {ext,* }}=\emptyset$ in Corollary 4.6. Then, there holds

$$
\left\{\sum_{D \in \mathcal{D}_{h}^{*}}\left(\eta_{\mathrm{DF}, D}+\eta_{\mathrm{R}, D}\right)^{2}\right\}^{\frac{1}{2}} \leq C \mid\left\|p-p_{h}\right\|_{\#},
$$

where the constant $C$ depends only on $d, \kappa_{\mathcal{S}}$, and $m$.

\subsection{Remarks and generalizations}

We conclude this section by several remarks and comments on generalizations.

Remark 5.7 (Unconditioned energy norm robustness with respect to discontinuous $a$ ). When the diffusion coefficient $a$ is piecewise constant on $\mathcal{D}_{h}$, when the harmonic averaging (2.5) has been used in Assumption 4.9, and when the flux has been reconstructed by (4.8), Theorem 5.1 implies a full robustness of the estimators of Theorem 4.5 with respect to the discontinuities in the diffusion coefficient $a$. No condition on the spatial distribution of the discontinuities in $a$ is necessary, whereas in the previous results $[12,43,23,1,17]$, a "monotonicity around vertices" condition or a similar assumption on the distribution of the diffusion coefficient was always necessary. When the diffusion coefficient $a$ is piecewise constant on $\mathcal{D}_{h}$ and when the harmonic averaging (2.5) has been used in Assumption 4.9, it can similarly be shown that the final upper bound of Theorem 5.5 can be changed from $C c_{a, D}^{-\frac{1}{2}} C_{a, \mathcal{T}_{D}}^{\frac{1}{2}}\left\|p-p_{h} \mid\right\|_{\mathcal{T}_{V_{D}}}$ to $C\left\||| p-p_{h}\right\|_{\mathcal{T}_{V_{D}}}$. Thus the same robustness result also holds for the construction of the equilibrated flux $\mathbf{t}_{h}$ by (4.11a)-(4.11b).

Remark 5.8 (Diffusion coefficient $a$ piecewise constant on $\mathcal{T}_{h}$ ). If $a$ is piecewise constant on $\mathcal{T}_{h}$ (whence the choice of the weights has no influence in Assumption 4.9) but harmonic averaging (2.5) has been used in order to define the diffusive flux $\mathbf{t}_{h}$ in (4.8) in the interior sides of each $D \in \mathcal{D}_{h}$, Theorem 5.1 gives

$$
\begin{aligned}
\eta_{\mathrm{DF}, D} & \leq C\left\|\mid p-p_{h}\right\| \|_{D}, \\
\eta_{\mathrm{R}, D} & \leq \tilde{C} c_{a, D}^{-\frac{1}{2}} C_{a, D}^{\frac{1}{2}}\|\mid\| p-p_{h}\|\|_{D} .
\end{aligned}
$$

Note in particular that one has the local efficiency directly on each dual volume $D \in \mathcal{D}_{h}$ and not on the patch $\mathcal{T}_{V_{D}}$ of the original simplicial elements sharing the vertex $V_{D}$, which is larger that $D$.

Similarly, the final upper bound of Theorem 5.5 changes in this case from $C c_{a, D}^{-\frac{1}{2}} C_{a, \mathcal{T}_{V_{D}}}^{\frac{1}{2}}\left\|p-p_{h} \mid\right\|_{\mathcal{T}_{V_{D}}}$ to $c_{a, D}^{-\frac{1}{2}} C_{a, D}^{\frac{1}{2}}\left\|p-p_{h}\right\|_{D}$.

Remark 5.9 (Unconditioned dual norm robustness). Note that Theorem 5.4 or Corollary 5.6 give full robustness with respect to the discontinuities in $a$ without any restriction on the distribution of the discontinuities (they can be piecewise constant on $\mathcal{T}_{h}$ or on $\mathcal{D}_{h}$ ) or type of averaging (both arithmetic and harmonic averaging is allowed) in Assumption 4.9. As a matter of fact, tensorvalued $\mathbf{A}$ can also be considered, cf. Remark 3.18, and the estimates are also robust with respect to the anisotropies in $\mathbf{A}$. However, these results are established in the dual norm $\|\mid \cdot\| \|_{\#}(2.11)$ and one only has global (and not local) efficiency.

\section{Application of the error estimate and efficiency results to the various numerical methods}

The a posteriori error estimates of Section 4 and their efficiency of Section 5 were presented generally, without any notion of a particular numerical scheme. We show in this section how the results of Section 4 and 5 can be applied to the different numerical methods of Section 3. 


\subsection{Finite volume-type methods}

The finite volume-type methods of Section 3 can be rewritten in the form of Assumption 4.9 as follows: taking into account the fact that $\nabla p_{h} \cdot \mathbf{n}_{D}$ have no jump across the boundary of $\partial D$, $D \in \mathcal{D}_{h}^{\text {int }}$, the weighted vertex-centered finite volume method of Definition 3.3 writes equivalently in the form of Assumption 4.9. The weights $\omega$ in Assumption 4.9 are then identical to those used in Definition 3.3. The weighted cell-centered finite volume method of Definition 3.1 can be written in the form of Assumption 4.9 using Corollary 3.14. Let the diffusion coefficient a piecewise constant on the mesh $\mathcal{T}_{h}$. Then the vertex-centered finite volume method of Definition 3.2 immediately writes equivalently in the form of Assumption 4.9, as in this case, both $a$ and $\nabla p_{h} \cdot \mathbf{n}_{D}$ have no jump across the boundary of $\partial D, D \in \mathcal{D}_{h}^{\text {int }}$ (the weights $\omega$ have no influence in this case).

\subsection{The finite element method}

The finite element method of Definition 3.5 writes equivalently in the form of Assumption 4.9 when both $a$ and $f$ are piecewise constant on the mesh $\mathcal{T}_{h}$, using Corollary 3.12 (in this case once again, both $a$ and $\nabla p_{h} \cdot \mathbf{n}_{D}$ have no jump across the boundary of $\partial D, D \in \mathcal{D}_{h}^{\text {int }}$, so the weights $\omega$ have no influence). In the case of general $f$, we proceed following [50]. Let $f_{h}$ be given by $(f, 1)_{K} /|K|$ on all $K \in \mathcal{T}_{h}$. We then have:

Theorem 6.1 (Guaranteed a posteriori error estimate for the finite element method). Let $p$ be the weak solution of problem (1.1a)-(1.1b), let $p_{h}$ be its finite element approximation given by (3.4), let $\tilde{p}$ be the weak solution of problem (1.1a)-(1.1b) with $f$ replaced by $f_{h}$, and let $\tilde{p}_{h}$ be its finite element approximation. Then

$$
\left\|\left|p-p_{h}\left\|\left|\leq\left\|\tilde{p}-\tilde{p}_{h}\right\|\right|+2\left\{\sum_{K \in \mathcal{T}_{h}} \eta_{\text {Osc }, K}^{2}\right\}^{\frac{1}{2}},\right.\right.\right.
$$

where

$$
\eta_{\mathrm{Osc}, K}:=C_{\mathrm{P}, K}^{\frac{1}{2}} \frac{h_{K}}{c_{a, K}^{\frac{1}{2}}}\left\|f-f_{K}\right\|_{K} \quad K \in \mathcal{T}_{h} .
$$

Proof. The triangle inequality implies

$$
\left|\left\|p-p_{h}\left|\left\|\leq\left|\left\|p-\tilde{p}\left|\|+\| \tilde{p}-\tilde{p}_{h}\right|||+|| \tilde{p}_{h}-p_{h}\right\|\right| .\right.\right.\right.\right.
$$

By the same reasoning as in the proof of Theorem 4.1, using the definitions of the weak solutions, and finally similarly as in the proof of Theorem 4.5,

$$
\begin{aligned}
\|p-\tilde{p}\| & =\sup _{\varphi \in H_{0}^{1}(\Omega),\|\varphi\|=1}(a \nabla(p-\tilde{p}), \nabla \varphi)=\sup _{\varphi \in H_{0}^{1}(\Omega),\|\varphi\|=1}\left(f-f_{h}, \varphi\right) \\
& \leq \sup _{\varphi \in H_{0}^{1}(\Omega),\|\varphi\| \|=1} \sum_{K \in \mathcal{T}_{h}}\left(f-f_{K}, \varphi-\varphi_{K}\right)_{K} \leq\left\{\sum_{K \in \mathcal{T}_{h}} \eta_{\mathrm{Osc}, K}^{2}\right\}^{\frac{1}{2}} .
\end{aligned}
$$

Estimating the term $\left\|\left|\tilde{p}_{h}-p_{h}\right|\right\|$ similarly in a discrete setting concludes the proof.

Theorem 6.1 is given in the energy norm setting; one proceeds completely similarly for the dual norm setting. 


\begin{tabular}{lllllllllll}
\hline Method & Def. & Dif. coef. $a$ & Weights & Constr. of $\mathbf{t}_{h}$ & $\mathcal{D}_{h}^{\text {int,* }}$ & $\mathcal{D}_{h}^{\text {ext,* }}$ & Efficiency & Rob. & Osc. \\
\hline WCCFV $/$ & $3.1 /$ & & & & & & & & & \\
WVCFV & 3.3 & pwc on $\mathcal{D}_{h}$ & $(2.5)$ & Sec. $4.3 .1-4.3 .3$ & $\mathcal{D}_{h}^{\text {int }}$ & $\mathcal{D}_{h}^{\text {ext }}$ & Thm. 5.1 & Yes & No \\
WCCFV & $3.1 /$ & & & & & & & & \\
WVCFV & 3.3 & pwc on $\mathcal{D}_{h}$ & $(2.5)$ & Sec. 4.3 .4 & $\mathcal{S}_{h}$ & $\emptyset$ & Thm. 5.5 & Yes & No \\
VCFV & 3.2 & pwc on $\mathcal{T}_{h}$ & - & Sec. $4.3 .1-4.3 .3$ & $\mathcal{D}_{h}^{\text {int }}$ & $\mathcal{D}_{h}^{\text {ext }}$ & Thm. 5.1 & No & No \\
VCFV & 3.2 & pwc on $\mathcal{T}_{h}$ & - & Sec. 4.3 .4 & $\mathcal{S}_{h}$ & $\emptyset$ & Thm. 5.5 & No & No \\
FE & 3.5 & pwc on $\mathcal{T}_{h}$ & - & Sec. 4.3.1-4.3.3 & $\mathcal{D}_{h}^{\text {int }}$ & $\mathcal{D}_{h}^{\text {ext }}$ & Thm. 5.1 & No & Yes \\
FE & 3.5 & pwc on $\mathcal{T}_{h}$ & - & Sec. 4.3 .4 & $\mathcal{S}_{h}$ & $\emptyset$ & Thm. 5.5 & No & Yes \\
HWFE & 3.6 & pwc on $\mathcal{D}_{h}$ & - & Sec. $4.3 .1-4.3 .3$ & $\mathcal{D}_{h}^{\text {int }}$ & $\mathcal{D}_{h}^{\text {ext }}$ & Thm. 5.1 & Yes & Yes \\
HWFE & 3.6 & pwc on $\mathcal{D}_{h}$ & - & Sec. 4.3 .4 & $\mathcal{S}_{h}$ & $\emptyset$ & Thm. 5.5 & Yes & Yes \\
\hline
\end{tabular}

Table 1: Recapitulative table of the different methods and results, energy norm estimates of Theorem 4.5

\begin{tabular}{lllllllllll}
\hline Method & Def. & Dif. coef. $a$ & Weights & Constr. of $\mathbf{t}_{h}$ & $\mathcal{D}_{h}^{\text {int,* }}$ & $\mathcal{D}_{h}^{\text {ext,* }}$ & Efficiency & Rob. & Osc. \\
\hline WCCFV $/$ & $3.1 /$ & & & & & & & & & \\
WVCFV & 3.3 & pwc on $\mathcal{D}_{h}$ & any & Sec. $4.3 .1-4.3 .3$ & $\mathcal{D}_{h}^{\text {int }}$ & $\mathcal{D}_{h}^{\text {ext }}$ & Thm. 5.4 & Yes & No \\
WCCFV & $3.1 /$ & & & & & & & & \\
WVCFV & 3.3 & pwc on $\mathcal{D}_{h}$ & any & Sec. 4.3 .4 & $\mathcal{S}_{h}$ & $\emptyset$ & Cor. 5.6 & Yes & No \\
VCFV & 3.2 & pwc on $\mathcal{T}_{h}$ & - & Sec. $4.3 .1-4.3 .3$ & $\mathcal{D}_{h}^{\text {int }}$ & $\mathcal{D}_{h}^{\text {ext }}$ & Thm. 5.4 & Yes & No \\
VCFV & 3.2 & pwc on $\mathcal{T}_{h}$ & - & Sec. 4.3 .4 & $\mathcal{S}_{h}$ & $\emptyset$ & Cor. 5.6 & Yes & No \\
FE & 3.5 & pwc on $\mathcal{T}_{h}$ & - & Sec. 4.3.1-4.3.3 & $\mathcal{D}_{h}^{\text {int }}$ & $\mathcal{D}_{h}^{\text {ext }}$ & Thm. 5.4 & Yes & Yes \\
FE & 3.5 & pwc on $\mathcal{T}_{h}$ & - & Sec. 4.3 .4 & $\mathcal{S}_{h}$ & $\emptyset$ & Cor. 5.6 & Yes & Yes \\
HWFE & 3.6 & pwc on $\mathcal{D}_{h}$ & - & Sec. $4.3 .1-4.3 .3$ & $\mathcal{D}_{h}^{\text {int }}$ & $\mathcal{D}_{h}^{\text {ext }}$ & Thm. 5.4 & Yes & Yes \\
HWFE & 3.6 & pwc on $\mathcal{D}_{h}$ & - & Sec. 4.3 .4 & $\mathcal{S}_{h}$ & $\emptyset$ & Cor. 5.6 & Yes & Yes \\
\hline
\end{tabular}

Table 2: Recapitulative table of the different methods and results, dual norm estimates of Corollary 4.6

\subsection{The finite element method with harmonic averaging}

When $a$ is piecewise constant on $\mathcal{D}_{h}$, the finite element method with harmonic averaging of Definition 3.6 leads to a scheme which is very close to the harmonic-weighted vertex-centered finite volume method of Definition 3.3. Indeed, for $d=2$, as $|D \cap K|=|K| / 3$ for $D \in \mathcal{D}_{h}$ associated with one of the vertices of $K \in \mathcal{T}_{h}$ for the meshes of Section 2.1, the coefficient $\left.\tilde{a}\right|_{K}$ from (3.5) is given by the harmonic averaging of the three values $a_{D}, a_{E}$, and $a_{F}$ that $a$ takes at the three dual volumes $D, E$, and $F$ associated with the three vertices of $K$. Consequently, for $f$ piecewise constant on $\mathcal{T}_{h},(3.6)$ gives (3.3) where $\{\{a\}\}_{\omega}$ is now the harmonic average of $a_{D}, a_{E}$, and $a_{F}$. To obtain a guaranteed estimate, one defines $\mathbf{t}_{h} \in \mathbf{R T N}\left(\mathcal{S}_{h}\right)$ by fixing $\mathbf{t}_{h} \cdot \mathbf{n}_{\sigma}$ by $-\tilde{a} \nabla p_{h} \cdot \mathbf{n}_{\sigma}$ on the boundary of $D \in \mathcal{D}_{h}^{\text {int }}$ and by (4.8) for the other sides of $\mathcal{S}_{h}$, while separating the oscillations in $f$ as in Theorem 6.1. Robustness can then be proved as in Theorem 5.1.

\subsection{Summary}

We summarize in Tables 1 and 2 the different theoretical results of the present paper. 


\section{Numerical experiments}

We present in this section the results of several numerical experiments. We will consider two different examples and present the results separately for the vertex-centered finite volume method of Definitions 3.2 and 3.3 and for the finite element method of Definition 3.5.

Example 7.1. We consider here a one-dimensional model problem

$$
\begin{aligned}
-p^{\prime \prime} & \left.=\pi^{2} \sin (\pi x) \quad \text { in }\right] 0,1[, \\
p & =0 \quad \text { in } 0,1 .
\end{aligned}
$$

The exact solution is smooth and given by $p(x)=\sin (\pi x)$.

Example 7.2. We consider here (1.1a)-(1.1b) with $\Omega=(-1,1) \times(-1,1)$, divided into four subdomains $\Omega_{i}$ along the Cartesian axes (the subregion $\{x>0, y>0\} \cap \Omega$ is denoted by $\Omega_{1}$ and the subsequent numbering is done counterclockwise) and the diffusion coefficient $a$ piecewise constant and equal to $a^{i}$ in $\Omega_{i}$. We set $f=0$. We suppose that the analytical solution writes

$$
p(r, \theta)=r^{\alpha}\left(a_{i} \sin (\alpha \theta)+b_{i} \cos (\alpha \theta)\right)
$$

in each $\Omega_{i}$. Here $(r, \theta)$ are the polar coordinates in $\Omega, a_{i}$ and $b_{i}$ are constants depending on $\Omega_{i}$, and $\alpha$ is a parameter. We assume Dirichlet boundary conditions given by this solution instead of the homogeneous ones (1.1b). This solution is continuous across the interfaces but only the normal component of its flux $\mathbf{u}=-\mathbf{S} \nabla p$ is continuous; it exhibits a singularity at the origin and it only belongs to $H^{1+\alpha}(\Omega)$. We consider two sets of the coefficients. In the first one, $a^{1}=a^{3}=5$, $a^{2}=a^{4}=1, \alpha=0.53544095$, and in the second one, $a^{1}=a^{3}=100, a^{2}=a^{4}=1, \alpha=0.12690207$. The corresponding values of $a_{i}, b_{i}$ can be found in, e.g., $[48,57]$.

Suppose a sequence of meshes $\mathcal{T}_{h}$, given by either a uniform or an adaptive refinement. We define the experimental order of convergence (e.o.c.) by

$$
\text { e.o.c. }:=\frac{\log \left(e_{N}\right)-\log \left(e_{N-1}\right)}{\frac{1}{d} \log \left|\mathcal{V}_{N-1}\right|-\frac{1}{d} \log \left|\mathcal{V}_{N}\right|} ;
$$

here $e_{N}$ is the error on the last mesh, $e_{N-1}$ is the error on the last but one mesh, and $\left|\mathcal{V}_{N}\right|$ and $\left|\mathcal{V}_{N-1}\right|$ denote the corresponding number of vertices; (we also recall that $d$ stands for the space dimension).

\subsection{Vertex-centered finite volume method}

We first present computational results for the vertex-centered finite volume method of Definition 3.2 and the weighted vertex-centered finite volume method of Definition 3.3.

\subsubsection{Energy norm estimates based on direct prescription and comparison with functional-type a posteriori estimates}

We consider here the vertex-centered finite volume method of Definition 3.2 for the problem of Example 7.1.

We consider a series of uniformly refined meshes and construct a one-dimensional equivalent of the equilibrated field $\mathbf{t}_{h}$ given by (4.8). The results for the estimates of Theorem 4.5 are reported in Figure 2. It turns out that in this one-dimensional setting, there actually holds $\left(\nabla \cdot \mathbf{t}_{h}, 1\right)_{K}=(f, 1)_{K}$ 

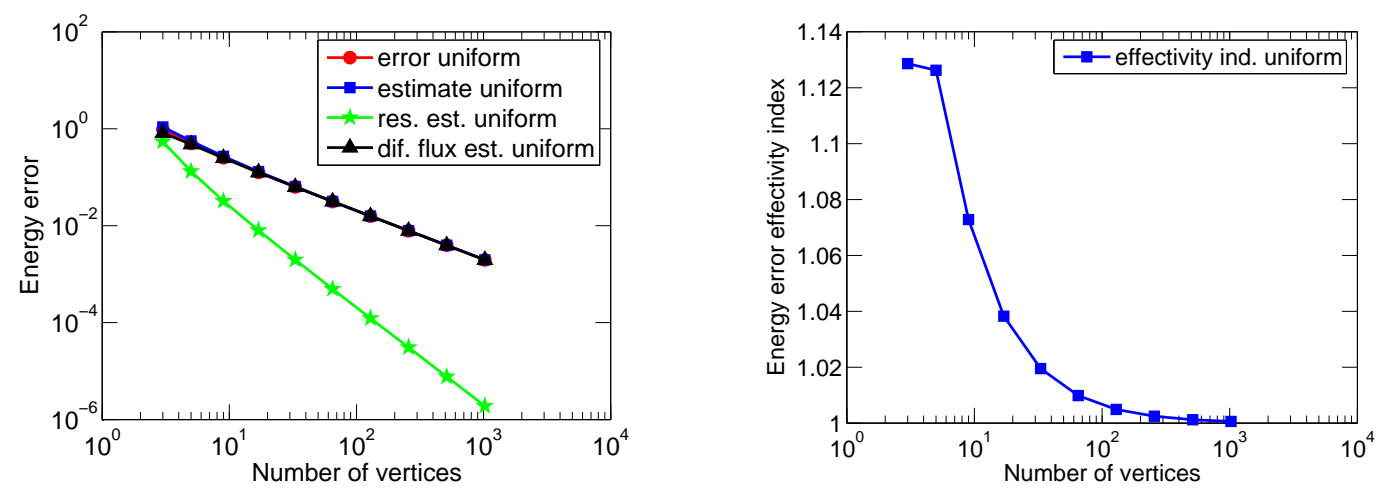

Figure 2: Estimated and actual energy error (left) and the corresponding effectivity index (right), vertex-centered finite volume method of Definition 3.2, Example (7.1), estimates by the direct prescription (4.8)
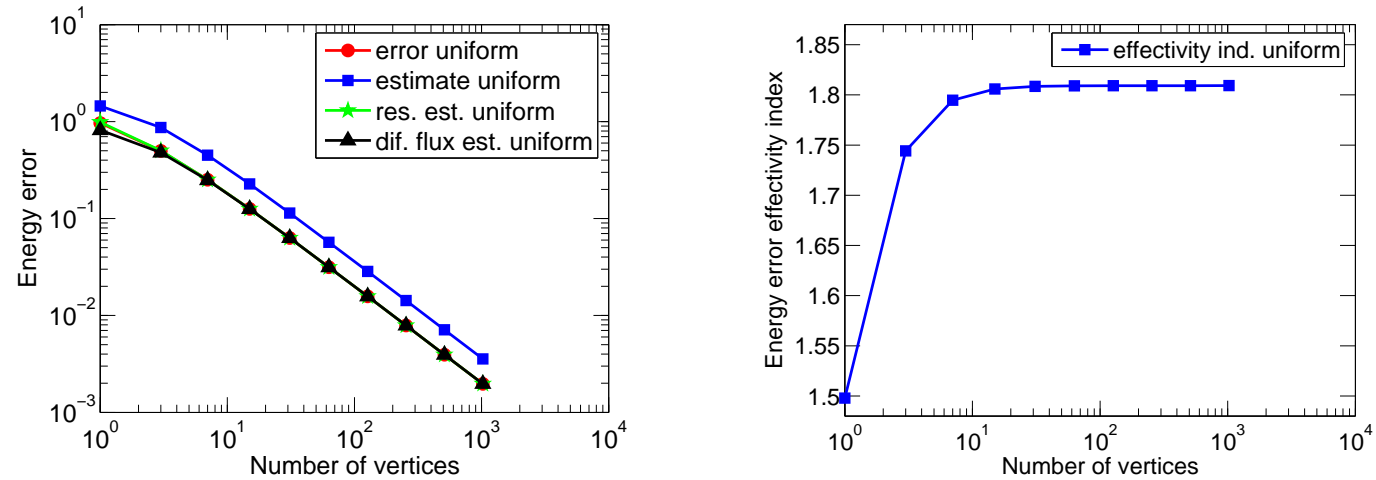

Figure 3: Estimated and actual energy error (left) and the corresponding effectivity index (right), vertex-centered finite volume method of Definition 3.2, Example (7.1), functional-type estimates (4.12) with the direct prescription (4.8)

for all elements $K$ of the fine mesh $\mathcal{S}_{h}$, in place of Assumption 4.4 (where such a mass balance is only supposed to be valid on patches $\mathcal{S}_{D}$ and not on each $K \in \mathcal{S}_{h}$ ). Consequently, the residual estimators $\eta_{\mathrm{R}, D}$ (4.3) represent a contribution of higher order and are only significant on coarsest meshes. We also observe asymptotic exactness in the right part of Figure 2. The e.o.c. is equal to 1.001 here.

In Figure 3, we present the results for the same model problem and the same construction of the equilibrated flux $\mathbf{t}_{h}$ (4.8), but with the estimate of Theorem 4.5 replaced by the functional-type estimate (4.12). The results of Figure 3 should be compared to those of Figure 2. The residual term in (4.12) features the diameter $h_{\Omega}$ of the whole domain $\Omega$ instead of $h_{D}$, the diameter of the dual volume $D$, as discussed in Remark 4.13. Consequently, this term is no more of the higher order $O\left(h^{2}\right)$ but only of order $O(h)$. In particular, the asymptotic exactness of the a posteriori error estimate is lost, but such an estimate is still perfectly usable.

\subsubsection{Robust energy norm estimates based on local minimization for dual mesh- aligned discontinuities and harmonic averaging}

We consider here the weighted vertex-centered finite volume method of Definition 3.3 for the 


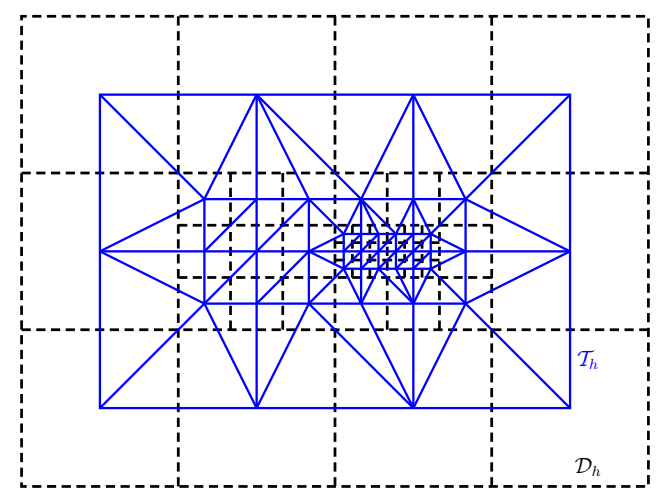

Figure 4: Example of a given nonmatching dual mesh $\mathcal{D}_{h}$ and the corresponding primal triangular mesh $\mathcal{T}_{h}$ for the harmonic-weighted vertex-centered finite volume method of Definition 3.3
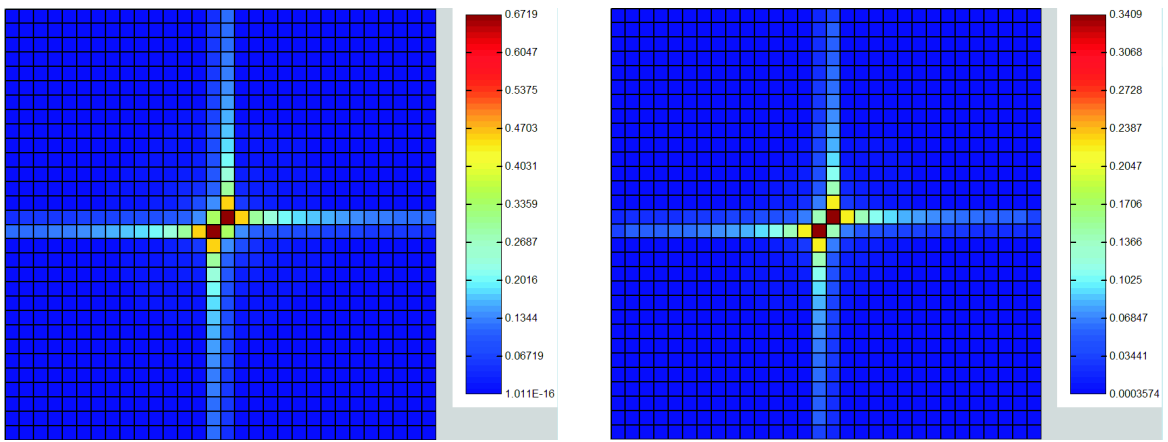

Figure 5: Estimated (left) and actual (right) energy error distribution on a uniformly refined mesh, harmonic-weighted vertex-centered finite volume method of Definition 3.3, Example 7.2 with $\alpha=0.535$, estimates by the direct prescription (4.8)

problem of Example 7.2.

In order to get robust a posteriori error estimates in the energy norm, we know from Theorem 5.1 that the diffusion coefficient $a$ has to be piecewise constant on $\mathcal{D}_{h}$. If, however, we would first construct a simplicial mesh $\mathcal{T}_{h}$ of $\Omega$ and then a dual grid $\mathcal{D}_{h}$ as in Section 2.1, it would be very difficult to keep the dual mesh aligned with the inhomogeneities, especially for adaptive refinement. A possible solution is to first define the dual mesh $\mathcal{D}_{h}$ and only then the primal one $\mathcal{T}_{h}$. On the resulting couple of grids $\mathcal{D}_{h}, \mathcal{T}_{h}$, we then use the weighted vertex-centered finite volume method of Definition 3.3 with the harmonic weights (2.5). Recall that on square grids (and their uniform refinements), this method is equivalent to the weighted cell-centered finite volume one, cf. Corollary 3.14, as well as to the finite difference one, cf. Remark 3.17. The advantage of the scheme (3.3) is that it can be used also when the original square grid has been locally refined (into a nonmatching grid) as in Figure 4. Note however that the symmetry of this scheme is then lost. We remark that the present methodology works also for the finite element method with harmonic averaging of Definition 3.6, which stays symmetric.

We in Figure 5 present the predicted and actual distribution of the error for $\alpha=0.535$ and uniform mesh refinement, using the estimators of Theorem 4.5 on the dual mesh $\mathcal{D}_{h}$ and with $\mathbf{t}_{h}$ given by (4.8) (the interpolation error on nonhomogeneous Dirichlet boundary conditions is neglected). A similar comparison, this time for adaptive mesh refinement and $\alpha=0.127$, is shown in Figure 6. A square cell of the original dual mesh is refined into 9 identical subsquares if the 

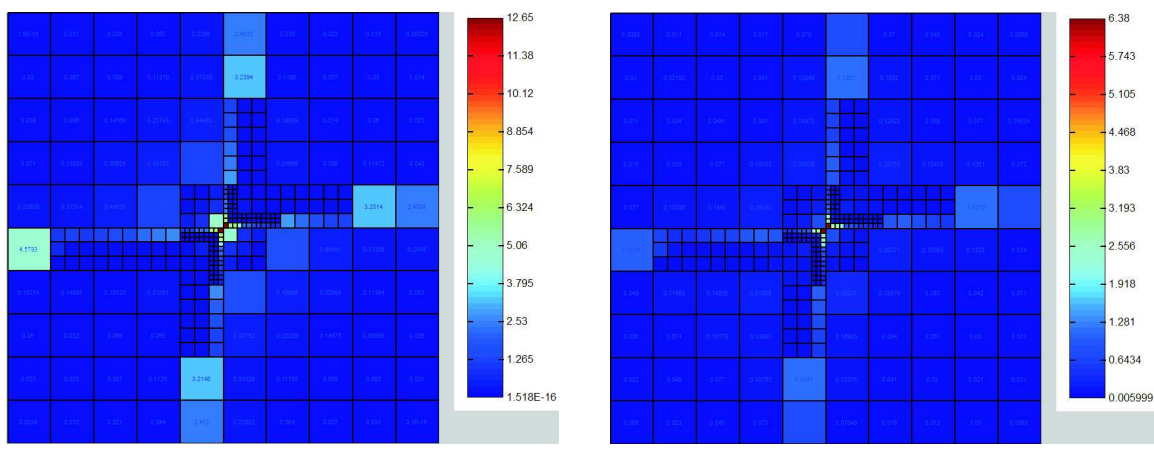

Figure 6: Estimated (left) and actual (right) energy error distribution on an adaptively refined mesh, harmonic-weighted vertex-centered finite volume method of Definition 3.3, Example 7.2 with $\alpha=0.127$, estimates by the direct prescription (4.8)
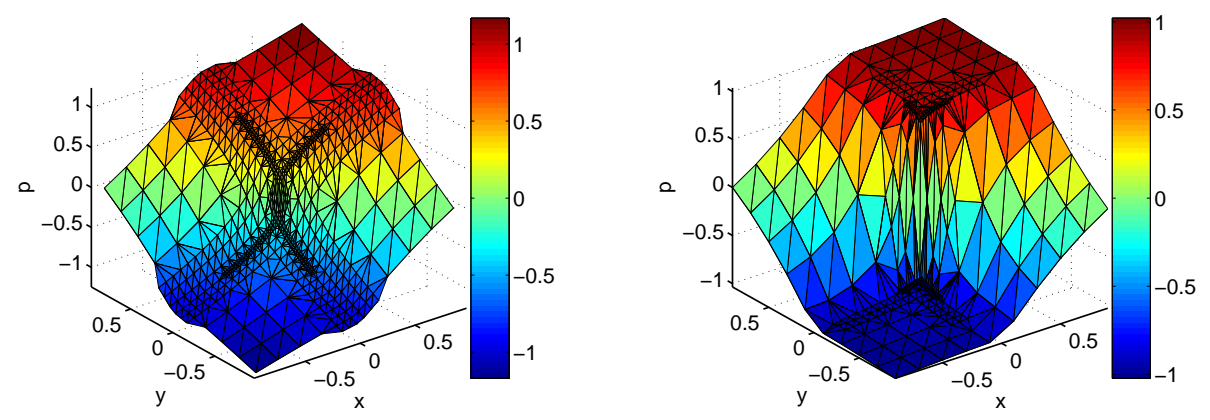

Figure 7: Approximate solutions on adaptively refined meshes, harmonic-weighted vertex-centered finite volume method of Definition 3.3, Example 7.2 with $\alpha=0.535$ (left) and $\alpha=0.127$ (right)

estimated energy error is greater than $50 \%$ of the maximum of the estimators. We can see that in both cases the predicted error distribution is excellent and that in particular, the singularity at the origin is well detected. These results clearly illustrate the robust local lower bound of Theorem 5.1. We finally in Figure 7 give examples of the approximate solutions on the adaptively refined meshes in both cases; the strength of the singularity in the second case is quite obvious.
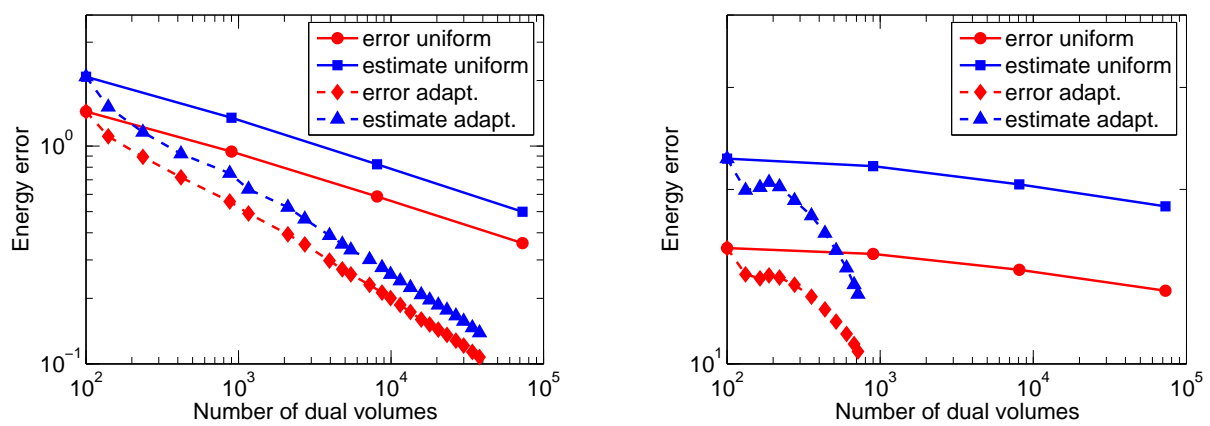

Figure 8: Estimated and actual energy errors, harmonic-weighted vertex-centered finite volume method of Definition 3.3, Example 7.2 with $\alpha=0.535$ (left) and $\alpha=0.127$ (right), estimates by the local minimization (4.9) 

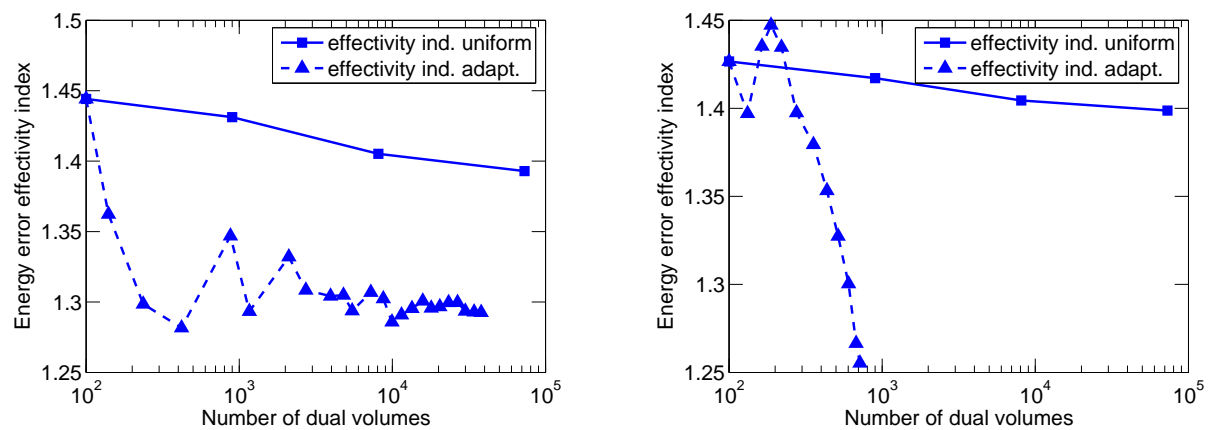

Figure 9: Energy error effectivity indices, harmonic-weighted vertex-centered finite volume method of Definition 3.3, Example 7.2 with $\alpha=0.535$ (left) and $\alpha=0.127$ (right), estimates by the local minimization (4.9)

Knowing precisely the error distribution and refining adaptively the meshes, the next step is to check whether this leads to an increased efficiency of the calculations. This is illustrated in Figure 8, from which it is evident that one can achieve a given precision with much fewer elements using adaptive mesh refinement based on our estimator. Here, the error in the energy norm (2.10) is approximated with a 7-point quadrature formula in each subtriangle $K \in \mathcal{S}_{D}$. In the code TALISMAN [60], which we use for numerical computations in this section, at most 9 levels of refinement can be used. This technical limitation is the reason why we in the adaptive case and for $\alpha=0.127$ only present results with at most 716 dual volumes - this maximal refinement level is achieved near the origin but the maximal error is still located there. For $\alpha=0.535$, the e.o.c. for uniform refinement was 0.449 and for the adaptive one 1.006. For $\alpha=0.127$, these values were respectively 0.0757 and 1.024. Following [7], the somewhat slower convergence rate for uniform refinement (compare with the finite element case below) in the energy norm is related to the fact that the diffusion coefficient $a$ is not aligned with the mesh $\mathcal{T}_{h}$ on which we reconstruct the approximate solution $p_{h}$.

Finally, in Figure 9, we give the effectivity indices (recall that these are defined as the ratio of the estimated and actual error) using the local minimization approach described in Section 4.3.3. We can clearly observe a confirmation of the robustness of our estimators: whereas the inhomogeneity ratio rises from 5 to 100, the effectivity indices stay at the level of 1.4 for uniform refinement and improve for adaptive refinement. Moreover, the local minimization of Section 4.3.3 allows for almost asymptotic exactness (the effectivity index is close to the optimal value of 1 ), and this even in the case of discontinuous coefficients and singular solutions.

\subsection{Finite element method}

We present here computational results for the finite element method of Definition 3.5, when applied to Example 7.2. The initial mesh consists of 24 right-angled triangles, conforming with the 4 subdomains $\Omega_{i}$.

\subsubsection{Energy norm estimates based on direct prescription and comparison with functional-type a posteriori estimates}

Here, we consider the case $\alpha=0.535$ and the equilibrated flux $\mathbf{t}_{h}$ given by the direct prescription (4.8); in (4.8), we take $\omega$ as the arithmetic averaging (2.4). We want to compare the results for 

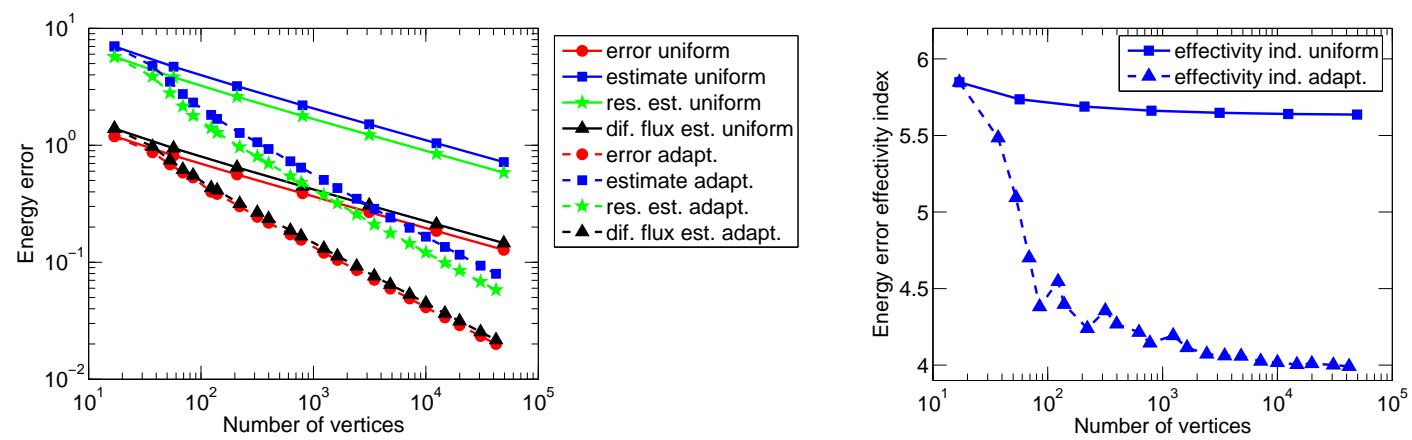

Figure 10: Estimated and actual energy error and the different estimators (left) and the corresponding effectivity index (right), finite element method of Definition 3.5, Example 7.2 with $\alpha=0.535$, estimates by the direct prescription (4.8)
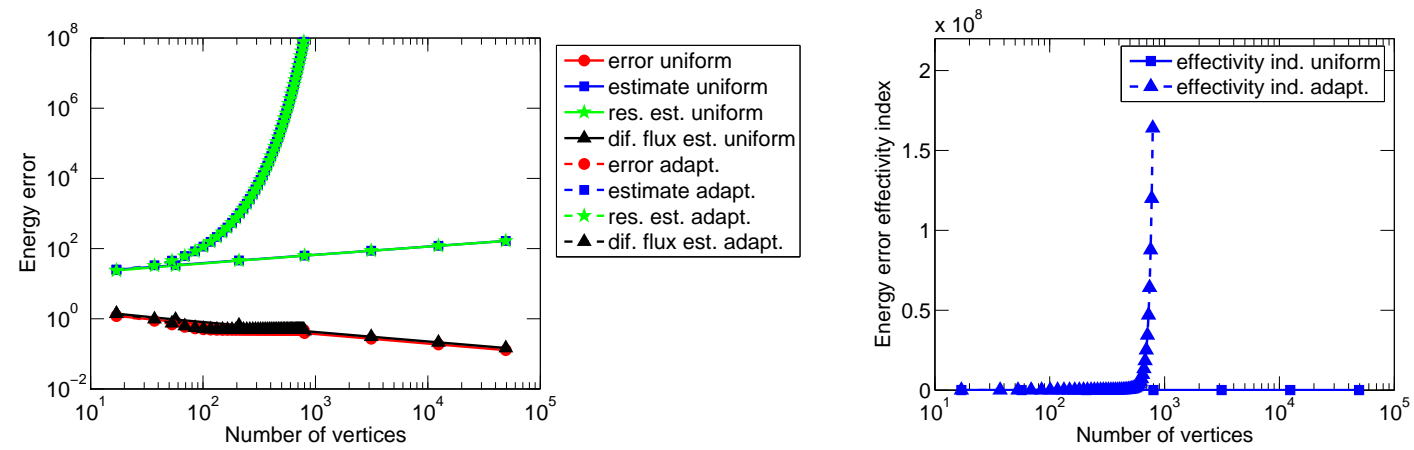

Figure 11: Estimated and actual energy error and the different estimators (left) and the corresponding effectivity index (right), finite element method of Definition 3.5, Example 7.2 with $\alpha=0.535$, functional-type estimates (4.12) with the direct prescription (4.8)

the estimate of Theorem 4.5, reported in Figure 10, and the corresponding results for the simple functional-type estimate (4.12), reported in Figure 11.

Recall that we did a similar comparison in Section 7.1.1. In the present section, the residual estimate of Theorem 4.5 is of the same order as the error, $O\left(h^{0.537}\right)$ for the uniform refinement, see Figure 10, left. It is in particular not superconvergent as it was in Section 7.1.1. The corresponding residual estimator of $(4.12)$ is $(1 / h)$-times bigger; this, however, means that it diverges in the present case. Such an effect is even more pronounced when using an adaptive refinement procedure, see Figure 11. The functional estimate (4.12), as discussed theoretically in Remark 4.13, is here not good at all. This observation confirms the general need to use some kind of global minimization for the functional-type estimates. Alternatively, if the equilibrated flux $\mathbf{t}_{h}$ is constructed by the local Neumann/Dirichlet mixed finite element problems (4.11a)-(4.11b), see the next section, the residual estimate gets zero. Then the estimate of Theorem 4.5 and the functional-type estimate (4.12) coincide.

\subsubsection{Energy norm estimates based on local Neumann/Dirichlet mixed finite element problems}

We present here the results for the energy error (2.10) estimates of Theorem 4.5 based on local Neumann/Dirichlet mixed finite element problems of Section 4.3.4. 

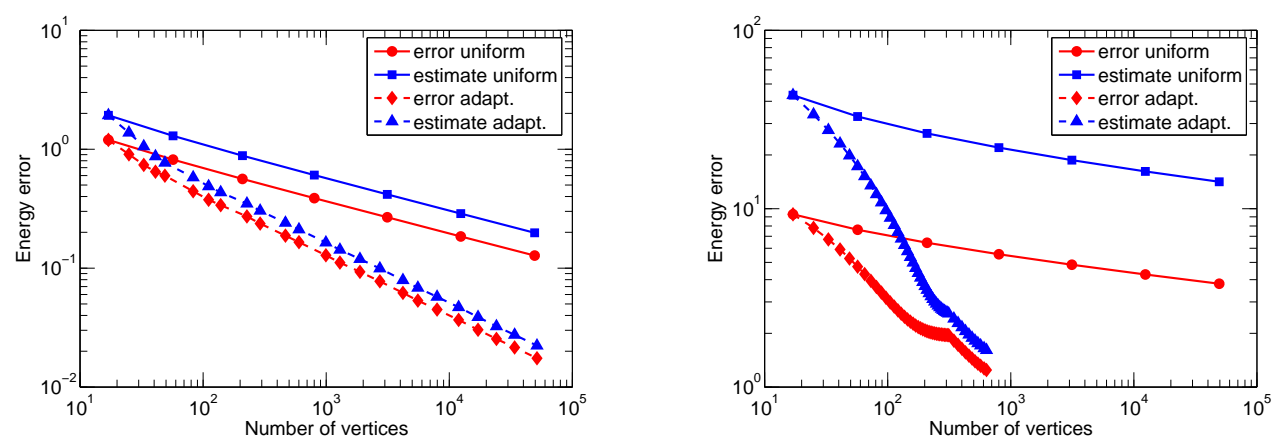

Figure 12: Estimated and actual energy errors, finite element method of Definition 3.5, Example 7.2 with $\alpha=0.535$ (left) and $\alpha=0.127$ (right), estimates by the local Neumann/Dirichlet mixed finite element problems (4.11a)-(4.11b)
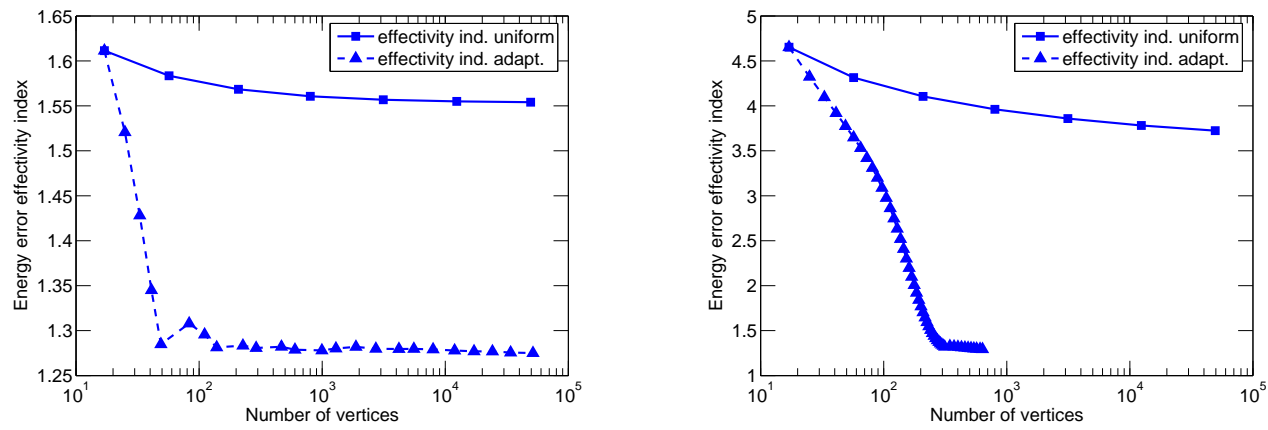

Figure 13: Energy error effectivity indices, finite element method of Definition 3.5, Example 7.2 with $\alpha=0.535$ (left) and $\alpha=0.127$ (right), estimates by the local Neumann/Dirichlet mixed finite element problems (4.11a)-(4.11b)

Figure 12 shows the estimated and actual energy errors. For $\alpha=0.535$, the e.o.c. for uniform refinement is 0.537 and for the adaptive one 0.999; for $\alpha=0.127$, these values are, respectively, 0.172 and 0.946 . This is fully in agreement with the smoothness of the weak solutions (recall that $\left.p \in H^{1+\alpha}(\Omega)\right)$ for the uniform refinement and shows optimal behavior of the adaptive refinement strategy. For $\alpha=0.127$, the adaptive refinement is stopped for roughly 700 elements as the diameter of the smallest triangles near the origin reaches $10^{-16}$, which is the computer double precision.

The corresponding effectivity indices are presented in Figure 13. As predicted by Theorem 5.5, we can observe in comparison with Figure 9 that the estimates are no more robust with respect to the discontinuities in $a$; while going from the contrast 5 in the discontinuity of $a$ in the left part of Figure 13 to the contrast 100 in the right part of Figure 13, the effectivity indices are no more of the same order as in Figure 9 but change (increase) abruptly. More precisely, the effectivity index is around 1.6 for $\alpha=0.535$ and 4.7 for $\alpha=0.127$ on the coarsest mesh, although it gets down to roughly 1.27 for adaptive mesh refinement for both tested values of the parameter $\alpha$. As seen from Figure 14, where we plot the estimated and actual energy error distribution the biggest overestimation appears around the center and the error distribution is no more predicted accurately (compare with Figures 5 and 6). It is the purpose of the two forthcoming sections to improve on these points. 

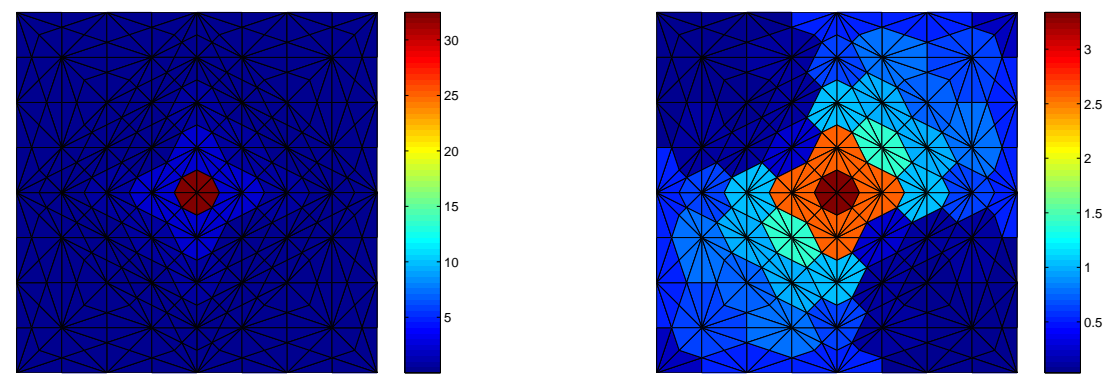

Figure 14: Estimated (left) and actual (right) energy error distribution, finite element method of Definition 3.5, Example 7.2 with $\alpha=0.127$, estimates by the local Neumann/Dirichlet mixed finite element problems (4.11a)-(4.11b)

\subsubsection{Robust dual norm estimates based on local Neumann/Dirichlet mixed finite element problems}

We present here the results for the dual error (2.11) estimates of Corollary 4.6 based on local Neumann/Dirichlet mixed finite element problems of Section 4.3.4.

Figure 15 reports the estimated and actual dual error; here "error up" means the computable upper bound on the dual error from (2.12), whereas "error down" means the computable lower bound from (2.12). In the dual error upper bound, for $\alpha=0.535$, the e.o.c. for uniform refinement is 0.539 and for the adaptive one 1.017; for $\alpha=0.127$, these values are, respectively, 0.195 and 1.109. In Figure 16, we then report the corresponding effectivity indices. We in particular see that the effectivity index in the dual error upper bound is independent of the jump in $a$ and close to the optimal value of 1 . The effectivity index in the dual error lower bound is, on the contrary, not independent of the jump in $a$. Remark that these observations are not in any contradiction with the theory. Recall that Corollaries 4.6 and 5.6 state that only the dual norm effectivity index, i.e., the ratio of the estimates of and of the dual error, is independent of the jump in the diffusion coefficient $a$ and bigger than equal to 1 . One conclusion from Figures 15 and 16 is that, in comparison with Figures 12 and 13, the nonrobustness has been shifted to the gap between the computable upper and lower bounds for the dual error. Finally, Figure 17 shows the predicted dual error distribution and actual dual upper bound error distribution which reveals excellent (note in particular that there is no gap in the scales of the figures, contrarily to the energy setting of Figure 14).

\subsubsection{Local refinements of individual dual volumes}

We finally come back shortly to the energy norm framework of Section 7.2.2. The idea is to solve the mixed finite element minimization problem (4.10) (or, equivalently, (4.11a)-(4.11b)) on a local refinement of the mesh $\mathcal{S}_{D}$ in individual dual volumes $D \in \mathcal{D}_{h}$, with the hope to decrease the error estimates in individual dual volumes, and, consequently, to improve the final error estimate value and distribution. The local refinement is driven by the quantity $\left\|a^{\frac{1}{2}} \nabla p_{h}+a^{-\frac{1}{2}} \mathbf{t}_{h}\right\|_{K}$ on each element $K$ of the local refinement of $\mathcal{S}_{D}$. We refine here only the central dual volume, as only in this dual volume the overestimation dependent on the jumps in $a$ occurs, see Figure 14. Figure 18 shows that this indeed enables to substantially decrease the effectivity indices (this figure is to be compared with Figure 13), although robustness is only achieved for $\alpha=0.535$; for $\alpha=0.127$, still an overestimation by a factor of 2.1 appears. Such a procedure also allows to predict much more precisely the error distribution, see the right part of Figure 19, to be compared with Figure 14. 

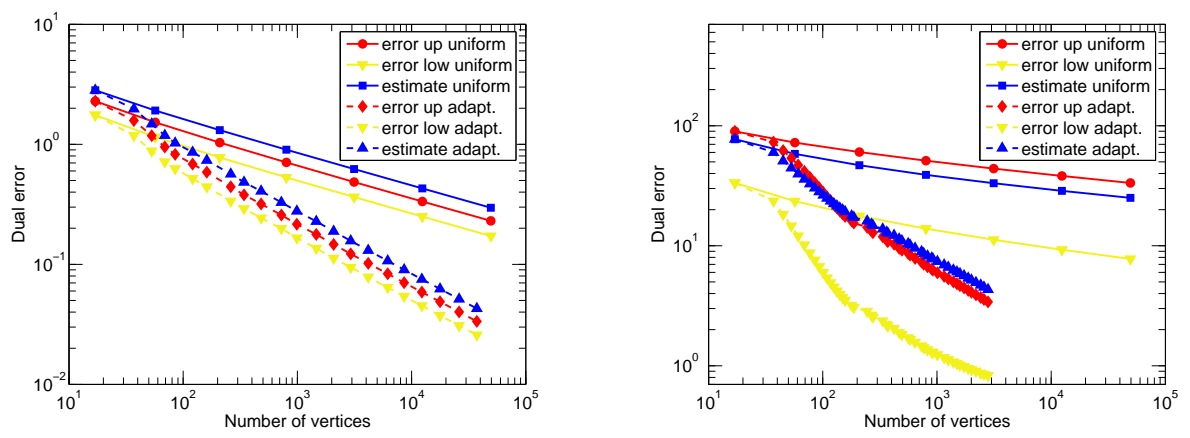

Figure 15: Estimated and actual dual errors, finite element method of Definition 3.5, Example 7.2 with $\alpha=0.535$ (left) and $\alpha=0.127$ (right), estimates by the local Neumann/Dirichlet mixed finite element problems (4.11a)-(4.11b)
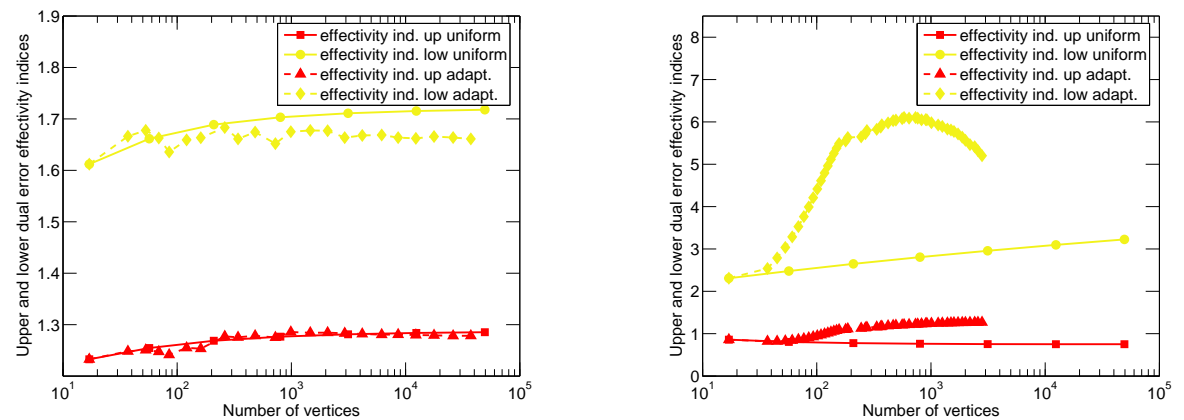

Figure 16: Dual error effectivity indices, finite element method of Definition 3.5, Example 7.2 with $\alpha=0.535$ (left) and $\alpha=0.127$ (right), estimates by the local Neumann/Dirichlet mixed finite element problems (4.11a)-(4.11b)

Consider those dual volumes where the error indicator gives large values. Consider the adaptive refinement of such dual volumes, as described above. We can also use such an independent refinement of each dual volume in other ways. We can, for example, include the obtained local refinement into the mesh of the entire domain, in replacement of the original elements. The results of such a procure are illustrated in the left part of Figure 19. In the present case, it allows to substantially improve the classical local refinement illustrated in the right part of Figure 13. Note that only two steps of the local refinement cycle on the global level allow to achieve the same precision as 49 steps in Section 7.2.2. Finally, the predicted and actual error distribution in the locally refined central dual volume is shown in Figure 20. It indicates that with the boundary conditions on $\partial D$ given by $-\left\{\left\{a \nabla p_{h} \cdot \mathbf{n}_{\sigma}\right\}\right\}$, which are embedded in the definition of the space $\operatorname{RTN}_{\mathrm{N}}\left(\mathcal{S}_{D}\right)$ of Section 4.3.4 and thus into the minimization (4.10), one cannot obtain a robust estimate and correct error distribution in the energy norm setting for the finite element method of Definition 3.5, even with such a local refinement of one dual volume. This indicates the nonlocality of the error distribution. Thus, in confirmation of the theory of Section 5, only the approaches of Sections 7.1.2 and 7.2.3 seem to give robust estimates (and correct error distribution). 

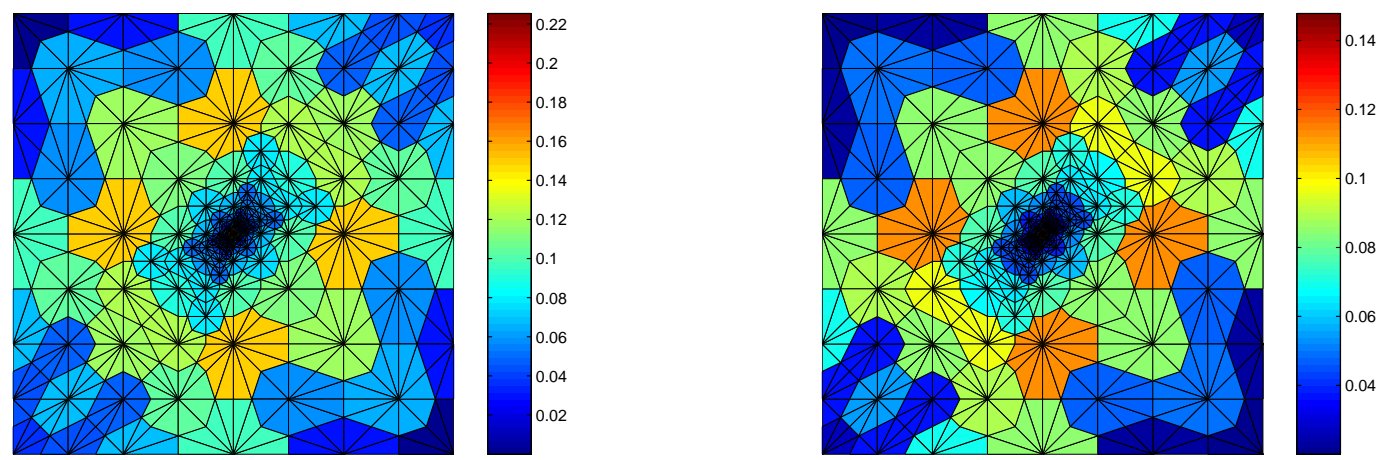

Figure 17: Estimated (left) and actual (right) dual error distribution, finite element method of Definition 3.5, Example 7.2 with $\alpha=0.535$, estimates by the local Neumann/Dirichlet mixed finite element problems (4.11a)-(4.11b)
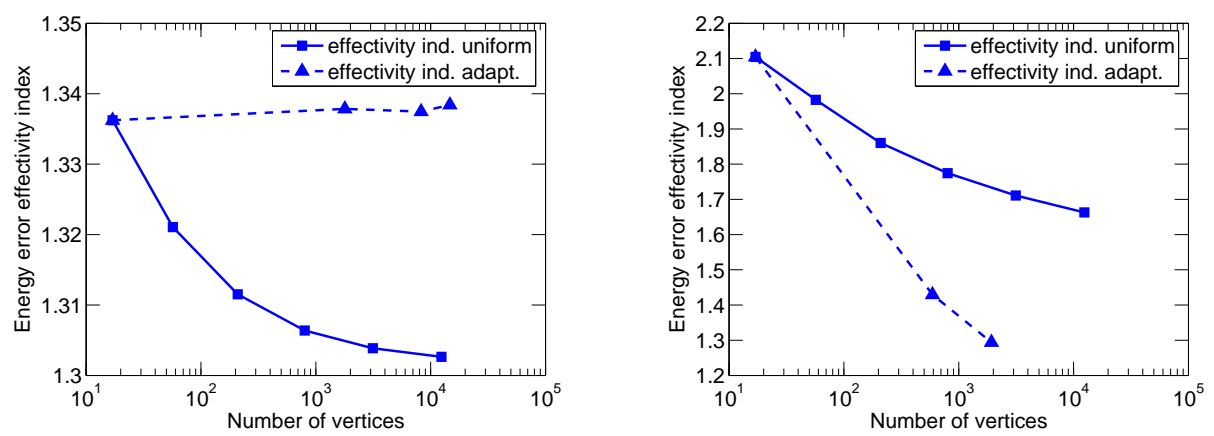

Figure 18: Energy error effectivity indices, finite element method of Definition 3.5, Example 7.2 with $\alpha=0.535$ (left) and $\alpha=0.127$ (right), estimates by local refinements of individual dual volumes of Section 7.2.4

\section{Conclusions}

We have focused in this paper on the pure diffusion model problem (1.1a)-(1.1b), with the importance on the possible jumps in the diffusion coefficient $a$. We have first, in Section 3, recalled some classical numerical methods for this problem and the close mutual relations between them. We have next proposed a unified a posteriori error estimate in Theorem 4.5 for the energy norm and in Corollary 4.6 for the dual norm. Both these results only suppose the existence of a very general equilibrated flux $\mathbf{t}_{h}$ (it only has to satisfy Assumption 4.4). We have then presented different practical constructions of an equilibrated flux $\mathbf{t}_{h}$, only based on the general form of a numerical scheme of Assumption 4.9.

We have next analyzed the efficiency and robustness of the proposed estimates. It turns out that in the setting of the energy norm (2.10), the harmonic averaging (2.5) has to be used in both the definition of the scheme and in the construction of the equilibrated flux (4.8), in order to obtain robustness with respect to the jumps in the diffusion coefficient $a$. No such a requirement is necessary in order to obtain robustness in the setting of the dual norm (2.11). We believe that the dual norm setting and the estimates of Corollary 4.6 are superior as more physical, see Remark 4.8.

We have introduced different constructions of the equilibrated flux $\mathbf{t}_{h}$. It is the solution of 

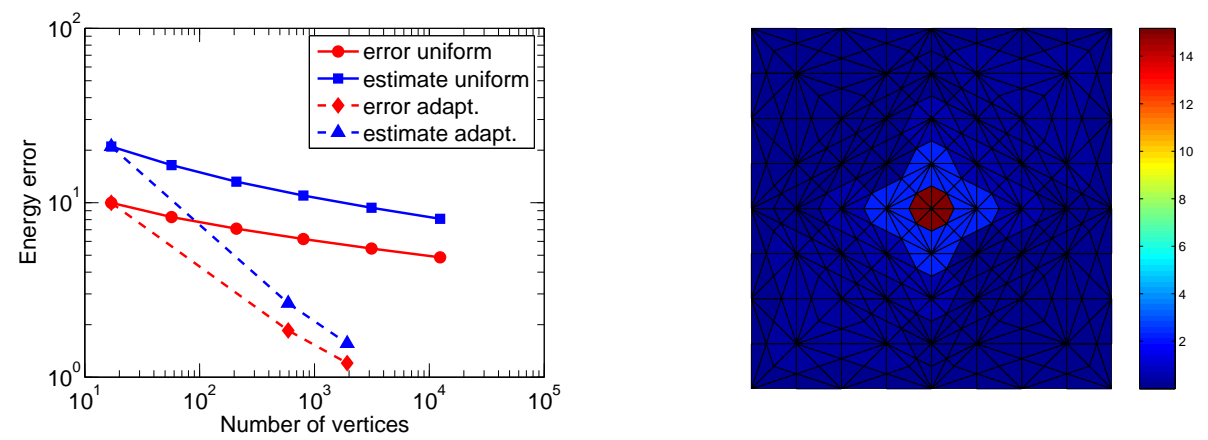

Figure 19: Estimated and actual energy error (left) and estimated energy error distribution (right), finite element method of Definition 3.5, Example 7.2 with $\alpha=0.127$, estimates by local refinements of individual dual volumes of Section 7.2.4
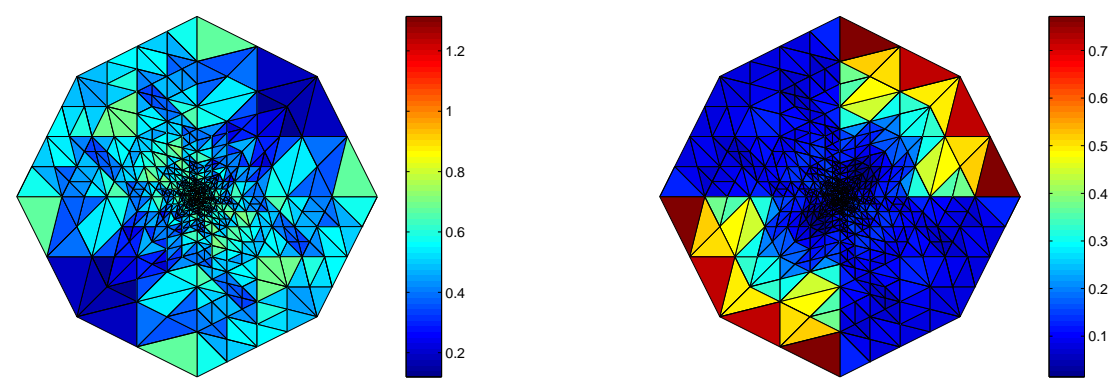

Figure 20: Estimated (left) and actual (right) energy error distribution on the locally refined central dual volume following Section 7.2.4, finite element method of Definition 3.5 Example 7.2 with $\alpha=0.127$

the local Neumann/Dirichlet problems by the mixed finite element method of Section 4.3.4 which seems in general to lead to the sharpest estimates. In order to avoid to implement the mixed finite element method and to solve the local problems (4.11a)-(4.11b), the approach of Section 4.3 .3 can be used while giving similar computational results (this approach is especially simple while taking $\left.\mathbf{t}_{h}\right|_{D}=\mathbf{t}_{2, D}$, i.e., $\left.\eta_{D}=\eta_{D}\left(\mathbf{t}_{2, D}\right)\right)$ instead of (4.9).

We finally remark that tensor diffusion coefficient $\mathbf{A}$ may be considered in (1.1a)-(1.1b) instead of the scalar one $a$. Many of the schemes of Section 3 apply in the same way as for scalar $a$, see Remark 3.18. We are not aware of a posteriori error estimates in the energy norm, robust with respect to the anisotropy in $\mathbf{A}$. The dual norm setting and the estimates of Corollary 4.6, however, lead to the robustness with respect to the anisotropy in $\mathbf{A}$, see Remark 5.9, in the same way as to the robustness with respect to the inhomogeneity.

\section{References}

[1] Ainsworth, M. Robust a posteriori error estimation for nonconforming finite element approximation. SIAM J. Numer. Anal. 42, 6 (2005), 2320-2341.

[2] Ainsworth, M. A synthesis of a posteriori error estimation techniques for conforming, nonconforming and discontinuous Galerkin finite element methods. In Recent advances in adaptive 
computation, vol. 383 of Contemp. Math. Amer. Math. Soc., Providence, RI, 2005, pp. 1-14.

[3] Ainsworth, M., And Oden, J. T. A posteriori error estimation in finite element analysis. Pure and Applied Mathematics (New York). Wiley-Interscience [John Wiley \& Sons], New York, 2000.

[4] Angermann, L. Balanced a posteriori error estimates for finite-volume type discretizations of convection-dominated elliptic problems. Computing 55, 4 (1995), 305-323.

[5] Arbogast, T., And Chen, Z. On the implementation of mixed methods as nonconforming methods for second-order elliptic problems. Math. Comp. 64, 211 (1995), 943-972.

[6] Arnold, D. N., And Brezzi, F. Mixed and nonconforming finite element methods: implementation, postprocessing and error estimates. RAIRO Modél. Math. Anal. Numér. 19, 1 (1985), 7-32.

[7] BABUŠKA, I. personnal communication. 2008.

[8] Babuška, I., And Rheinboldt, W. C. Error estimates for adaptive finite element computations. SIAM J. Numer. Anal. 15, 4 (1978), 736-754.

[9] Bank, R. E., and Rose, D. J. Some error estimates for the box method. SIAM J. Numer. Anal. 24, 4 (1987), 777-787.

[10] Bank, R. E., And Weiser, A. Some a posteriori error estimators for elliptic partial differential equations. Math. Comp. 44, 170 (1985), 283-301.

[11] Bebendorf, M. A note on the Poincaré inequality for convex domains. Z. Anal. Anwendungen 22, 4 (2003), 751-756.

[12] Bernardi, C., and Verfürth, R. Adaptive finite element methods for elliptic equations with non-smooth coefficients. Numer. Math. 85, 4 (2000), 579-608.

[13] Braess, D. Finite elements, third ed. Cambridge University Press, Cambridge, 2007. Theory, fast solvers, and applications in elasticity theory, Translated from the German by Larry L. Schumaker.

[14] Braess, D., Pillwein, V., And Schöberl, J. Equilibrated residual error estimates are p-robust. Comput. Methods Appl. Mech. Engrg. 198, 13-14 (2009), 1189-1197.

[15] Braess, D., ANd SchöBerl, J. Equilibrated residual error estimator for edge elements. Math. Comp. 77, 262 (2008), 651-672.

[16] Brezzi, F., And Fortin, M. Mixed and hybrid finite element methods, vol. 15 of Springer Series in Computational Mathematics. Springer-Verlag, New York, 1991.

[17] CAI, Z., AND Zhang, S. Recovery-based error estimator for interface problems: conforming linear elements. SIAM J. Numer. Anal. 47, 3 (2009), 2132-2156.

[18] Carstensen, C., And Funken, S. A. Constants in Clément-interpolation error and residual based a posteriori error estimates in finite element methods. East-West J. Numer. Math. 8, 3 (2000), 153-175. 
[19] Chaillou, A. L., And Suri, M. Computable error estimators for the approximation of nonlinear problems by linearized models. Comput. Methods Appl. Mech. Engrg. 196, 1-3 (2006), 210-224.

[20] Challlou, A. L., And Suri, M. A posteriori estimation of the linearization error for strongly monotone nonlinear operators. J. Comput. Appl. Math. 205, 1 (2007), 72-87.

[21] Cheddadi, I., Fučík, R., Prieto, M. I., and Vohralík, M. Computable a posteriori error estimates in the finite element method based on its local conservativity: improvements using local minimization. ESAIM Proc. 24 (2008), 77-96.

[22] Cheddadi, I., Fučík, R., Prieto, M. I., and Vohralík, M. Guaranteed and robust a posteriori error estimates for singularly perturbed reaction-diffusion problems. M2AN Math. Model. Numer. Anal. 43, 5 (2009), 867-888.

[23] Chen, Z., And Dai, S. On the efficiency of adaptive finite element methods for elliptic problems with discontinuous coefficients. SIAM J. Sci. Comput. 24, 2 (2002), 443-462.

[24] Destuynder, P., And Métivet, B. Explicit error bounds in a conforming finite element method. Math. Comp. 68, 228 (1999), 1379-1396.

[25] Dörfler, W., And Wilderotter, O. An adaptive finite element method for a linear elliptic equation with variable coefficients. ZAMM Z. Angew. Math. Mech. 80, 7 (2000), $481-491$.

[26] El Alaoui, L., Ern, A., And Vohralík, M. Guaranteed and robust a posteriori error estimates and balancing discretization and linearization errors for monotone nonlinear problems. Comput. Methods Appl. Mech. Engrg. (2010). DOI 10.1016/j.cma.2010.03.024.

[27] Ern, A., Stephansen, A. F., and Vohralík, M. Guaranteed and robust discontinuous Galerkin a posteriori error estimates for convection-diffusion-reaction problems. J. Comput. Appl. Math. 234, 1 (2010), 114-130.

[28] ERn, A., ANd Vohralík, M. Flux reconstruction and a posteriori error estimation for discontinuous Galerkin methods on general nonmatching grids. C. R. Math. Acad. Sci. Paris 347 (2009), 441-444.

[29] Eymard, R., Gallouët, T., and Herbin, R. Finite volume methods. In Handbook of Numerical Analysis, Vol. VII. North-Holland, Amsterdam, 2000, pp. 713-1020.

[30] Eymard, R., Hilhorst, D., and Vohralík, M. A combined finite volume-nonconforming/mixed-hybrid finite element scheme for degenerate parabolic problems. Numer. Math. 105, 1 (2006), 73-131.

[31] Fierro, F., ANd Veeser, A. A posteriori error estimators, gradient recovery by averaging, and superconvergence. Numer. Math. 103, 2 (2006), 267-298.

[32] Hannukainen, A., Stenberg, R., and Vohralík, M. A unified framework for a posteriori error estimation for the Stokes problem. Preprint R10016, Laboratoire Jacques-Louis Lions \& HAL Preprint 00470131, submitted for publication, 2010.

[33] Haslinger, J., And HlaváČEK, I. Convergence of a finite element method based on the dual variational formulation. Apl. Mat. 21, 1 (1976), 43-65. 
[34] HlaváČek, I., Haslinger, J., NeČAs, J., And LovíšEk, J. Solution of variational inequalities in mechanics, vol. 66 of Applied Mathematical Sciences. Springer-Verlag, New York, 1988. Translated from the Slovak by J. Jarník.

[35] Konotov, S. Two-sided a posteriori error estimates for linear elliptic problems with mixed boundary conditions. Appl. Math. 52, 3 (2007), 235-249.

[36] Ladevèze, P. Comparaison de modèles de milieux continus. Ph.D. thesis, Université Pierre et Marie Curie (Paris 6), 1975.

[37] Ladevèze, P., And Leguillon, D. Error estimate procedure in the finite element method and applications. SIAM J. Numer. Anal. 20, 3 (1983), 485-509.

[38] Letniowski, F. W. Three-dimensional Delaunay triangulations for finite element approximations to a second-order diffusion operator. SIAM J. Sci. Statist. Comput. 13, 3 (1992), $765-770$.

[39] Luce, R., And Wohlmuth, B. I. A local a posteriori error estimator based on equilibrated fluxes. SIAM J. Numer. Anal. 42, 4 (2004), 1394-1414.

[40] NeČas, J., And HlaváČEK, I. Mathematical theory of elastic and elasto-plastic bodies: an introduction, vol. 3 of Studies in Applied Mechanics. Elsevier Scientific Publishing Co., Amsterdam, 1980.

[41] NeittaAnmäki, P., And Repin, S. Reliable methods for computer simulation, vol. 33 of Studies in Mathematics and its Applications. Elsevier Science B.V., Amsterdam, 2004. Error control and a posteriori estimates.

[42] Payne, L. E., And Weinberger, H. F. An optimal Poincaré inequality for convex domains. Arch. Rational Mech. Anal. 5 (1960), 286-292.

[43] Petzoldt, M. A posteriori error estimators for elliptic equations with discontinuous coefficients. Adv. Comput. Math. 16, 1 (2002), 47-75.

[44] Prager, W., And Synge, J. L. Approximations in elasticity based on the concept of function space. Quart. Appl. Math. 5 (1947), 241-269.

[45] Putti, M., And Cordes, C. Finite element approximation of the diffusion operator on tetrahedra. SIAM J. Sci. Comput. 19, 4 (1998), 1154-1168.

[46] Repin, S. I. A posteriori error estimation for nonlinear variational problems by duality theory. Zap. Nauchn. Sem. S.-Peterburg. Otdel. Mat. Inst. Steklov. (POMI) 243, Kraev. Zadachi Mat. Fiz. i Smezh. Vopr. Teor. Funktsii. 28 (1997), 201-214, 342.

[47] Repin, S. I. A posteriori estimates for partial differential equations, vol. 4 of Radon Series on Computational and Applied Mathematics. Walter de Gruyter GmbH \& Co. KG, Berlin, 2008 .

[48] Rivière, B., Wheeler, M. F., And Banas, K. Part II. Discontinuous Galerkin method applied to single phase flow in porous media. Comput. Geosci. 4, 4 (2000), 337-349.

[49] Roberts, J. E., And Thomas, J.-M. Mixed and hybrid methods. In Handbook of Numerical Analysis, Vol. II. North-Holland, Amsterdam, 1991, pp. 523-639. 
[50] SchÖBERL, J. personnal communication. 2009.

[51] Synge, J. L. The hypercircle in mathematical physics: a method for the approximate solution of boundary value problems. Cambridge University Press, New York, 1957.

[52] VACEK, J. Dual variational principles for an elliptic partial differential equation. Apl. Mat. 21, 1 (1976), 5-27.

[53] Vejchodský, T. Guaranteed and locally computable a posteriori error estimate. IMA J. Numer. Anal. 26, 3 (2006), 525-540.

[54] VerfüRTH, R. A review of a posteriori error estimation and adaptive mesh-refinement techniques. Teubner-Wiley, Stuttgart, 1996.

[55] Verfürth, R. Robust a posteriori error estimates for stationary convection-diffusion equations. SIAM J. Numer. Anal. 43, 4 (2005), 1766-1782.

[56] Vohralík, M. On the discrete Poincaré-Friedrichs inequalities for nonconforming approximations of the Sobolev space $H^{1}$. Numer. Funct. Anal. Optim. 26, 7-8 (2005), 925-952.

[57] Vohralík, M. A posteriori error estimates for lowest-order mixed finite element discretizations of convection-diffusion-reaction equations. SIAM J. Numer. Anal. 45, 4 (2007), 15701599 .

[58] Vohralík, M. A posteriori error estimation in the conforming finite element method based on its local conservativity and using local minimization. C. R. Math. Acad. Sci. Paris 346, 11-12 (2008), 687-690.

[59] Vohralík, M. Two types of guaranteed (and robust) a posteriori estimates for finite volume methods. In Finite Volumes for Complex Applications V. ISTE and John Wiley \& Sons, London, UK and Hoboken, USA, 2008, pp. 649-656.

[60] Vohralík, M., and Ramarosy, N. Talisman, a finite volume-finite element tool for numerical simulation of subsurface flow and contaminant transport with a posteriori error control and adaptive mesh refinement. Presentation and User guide. Tech. rep., HydroExpert, 53 rue Charles Frérot, 94250 Gentilly, France, www.hydroexpert.com, 2007.

[61] Xu, J., Zhu, Y., And Zou, Q. New adaptive finite volume methods and convergence analysis. Preprint AM296, Mathematics Department, Penn State, 2006.

[62] Zienkiewicz, O. C., And Zhu, J. Z. A simple error estimator and adaptive procedure for practical engineering analysis. Internat. J. Numer. Methods Engrg. 24, 2 (1987), 337-357. 